\title{
An unconstrained integral approximation of large sliding frictional contact between deformable solids
}

\author{
Poulios, Konstantinos; Renard, Yves
}

Published in:

Computers \& Structures

Link to article, DOI:

10.1016/j.compstruc.2015.02.027

Publication date:

2015

Document Version

Peer reviewed version

Link back to DTU Orbit

Citation (APA):

Poulios, K., \& Renard, Y. (2015). An unconstrained integral approximation of large sliding frictional contact between deformable solids. Computers \& Structures, 153, 75-90.

https://doi.org/10.1016/j.compstruc.2015.02.027

\section{General rights}

Copyright and moral rights for the publications made accessible in the public portal are retained by the authors and/or other copyright owners and it is a condition of accessing publications that users recognise and abide by the legal requirements associated with these rights.

- Users may download and print one copy of any publication from the public portal for the purpose of private study or research.

- You may not further distribute the material or use it for any profit-making activity or commercial gain

- You may freely distribute the URL identifying the publication in the public portal 


\title{
An unconstrained integral approximation of large sliding frictional contact between deformable solids
}

\author{
Konstantinos Poulios * $\quad$ Yves Renard $^{\dagger}$
}

March 7, 2015

\begin{abstract}
A new integral approximation of frictional contact problems under large deformations is presented. Impenetrability, friction and the relevant complementarity conditions are expressed through a non-smooth equation, considered in the continuous setting. A weak formulation of this non-smooth complementarity equation is discretized through a standard Galerkin procedure, is linearized consistently and incorporated in a generalized Newton solution process. The resulting integral handling of contact and friction complementarity conditions, previously implemented for small deformations only, is extended in the present paper to large deformations. In total, the proposed method is relatively simple to implement, while its robustness is illustrated through numerical examples.
\end{abstract}

Keywords: frictional contact, large sliding contact, finite element, non-smooth complementarity equation, generalized Newton.

\section{Introduction}

Numerical modeling of frictional contact between solids undergoing large deformations is a challenging task, mainly because it involves complex geometrical and mechanical quantities that depend on an a priori unknown mapping between the surfaces in contact. Despite the multitude of very elaborate methods, proposed for solving this problem, there is an ongoing effort for improving the performance and robustness of currently available algorithms but also for simplifying the corresponding software implementations.

Although an exhaustive review of the field would be difficult, most methods that can represent frictional contact between deformable bodies under large deformations fall under the following categories:

1) Node-to-segment methods [18], possibly enhanced with smoothing techniques [23].

2) Mortar methods [10], possibly in combination with definition of contact segments [24].

3) Contact domain methods [12, 20, 32] and intermediate surface methods [19].

*Department of Mechanical Engineering, Technical University of Denmark, Nils Koppels Allé, Building 404, 2800 Kgs. Lyngby, Denmark. email: kopo@mek.dtu.dk

${ }^{\dagger}$ Université de Lyon, CNRS, INSA-Lyon, ICJ UMR5208, LaMCoS UMR5259, F-69621, Villeurbanne, France. email: yves.renard@insa-lyon.fr 
Node-to-segment and mortar methods normally represent asymmetric formulations, in the sense that the surfaces in contact are treated differently by distinguishing between a master (or mortar or target) and a slave (or non-mortar or contactor) surface. On the contrary, contact domain and intermediate surface methods are by their nature symmetric, hence they are intrinsically applicable to cases like self-contact and simultaneous contact between more than two solids, where asymmetric formulations usually require special treatment. Their main drawback is that for an arbitrary three-dimensional geometry, triangulation of a contact domain or definition of an intermediate surface can be complex or not even guaranteed. The here proposed approximation is comparable to the mortar methods presented in [10] and [28], in the sense that it relies on the available discretization of the slave surface for performing numerical integration of all relevant contact terms.

Especially in the context of large deformations, the mapping between the surfaces in contact is an important component in formulating a numerical approximation of the frictional contact problem. Node-to-segment methods traditionally map points of the slave surface to their closest point projection onto the master surface. Hence, the master surface normals govern the definition of a gap function and its kinematics, presented in detail in [18]. This classical mapping will in the following be simply referred to as the projection strategy. A different approach for defining a mapping between the slave and master surfaces is to find the closest intersection with the master surface along the slave surface normals. This mapping which is more common for mortar methods, will in the following be referred to as the ray-tracing strategy.

Specifically referring to mortar methods for contact under large deformations, the formulations presented for instance in [10, 16] employ the classical projection approach, while [24, 33, 28, 11] present formulations that rely on the ray-tracing strategy. Other occurrences of the ray-tracing strategy can be found in [29] as a contact search method as well as in connection to contact problems under small deformations, for instance, in the segment-to-segment approach presented in [34] and in the Nitsche formulation introduced in [31. The approach followed in the present paper relies on the ray-tracing strategy for deriving a contact formulation not depending on the curvature of the master surface with an optimality system which is not discontinuous across mesh edges or vertices, without requiring any smoothing technique.

Another crucial component in the numerical treatment of contact problems is the method for enforcing contact and friction conditions. Apart from the classical penalty method with its well known accuracy limitations, alternative approaches introduce multipliers for dealing with inequality and complementarity constraints, for instance, in the context of an augmented Lagrangian or interior point formulation. The unknown displacement and multiplier fields can be determined iteratively based on different fixed point techniques [17, including the very popular Uzawa method proposed in [26]. Alternatively, the generalized (or semi-smooth) Newton algorithm can be applied to the full system of equations including both displacements and multipliers [1, 25] or equivalently the solution can be based on a primal-dual active set strategy [15].

One implication related to transitioning from penalty to Lagrange multiplier based formulations in the context of mortar methods, concerns the discretization of complementarity conditions. As discussed in detail in [9], penalty formulations in mortar methods permit an integral enforcement of the contact complementarity condition by evaluating it at quadrature points. On the contrary, Lagrange multiplier based mortar methods evaluate contact and friction complementarity conditions with respect to nodal values of the Lagrange multiplier and weighted gap or slip values [9, 11]. This kind of discrete enforcement of complementarity conditions has two important consequences: 
a) The Lagrange multiplier field can only be approximated through Lagrange elements, so that finite element nodal values can be used in the evaluation of complementarity conditions.

b) Each finite element node of the Lagrange multiplier can be associated to either the active or the inactive set of a complementarity condition. Intermediate states cannot be approximated adequately even if the number of quadrature points is increased.

The main characteristic of the here proposed method is an integral approximation of the contact and friction complementarity conditions in the context of an augmented Lagrangian formulation. Impenetrability, Coulomb friction stress threshold and the corresponding complementarity conditions are expressed as a semi-smooth equation in the continuous space, incorporated in the weak formulation of the problem and discretized according to a standard Galerkin procedure. Very few occurrences of such an integral approach can be found in the computational contact mechanics literature. To the authors' knowledge, the fundamental idea of an integral enforcement of a complementarity equation, capturing all contact and friction conditions, was originally proposed in [8]. Nevertheless, the actual implementation included in [8] is a nodal one, while the implementation found in [21] relies on a quadrature-point-wise definition of the unknown Lagrange multipliers. In the current work, similar to [17] and [25], the Lagrange multiplier field is approximated on a finite element space and contact and friction conditions are enforced in a weak sense. Unlike references [17] and [25] however, the present work is not limited to the small deformations setting.

The proposed method, apart from representing a mathematically rigorous approximation, has the advantage that it does not require to prescribe constraints on the discretized Lagrange multiplier any longer, like for instance negativity of the contact pressure and a Coulomb threshold on the friction stress. The full set of contact, friction and complementarity constraints are already included in the weak formulation. As an interesting consequence, the formulation is independent of the finite element methods chosen to approximate the displacements and Lagrange multiplier fields. This characteristic offers the possibility of combining the proposed formulation, in the future, with less common approximations than the classical Lagrange finite elements, like for instance $C^{1}$ continuous Hermite and enriched finite elements as well as isogeometric analysis approximations of contact under large deformations, like [6, 27].

The paper is organized in nine sections. Following this introduction, Section 2 presents the basic problem setting and some notation conventions. Section 3 provides a comparison between the classical projection and ray-tracing strategies. Section 4 presents the weak formulation of the frictionless case along with some comments about discontinuities in the contexts of the projection and ray-tracing strategies. Sections 5 and 6 respectively describe the proposed weak formulation and finite element approximation for frictional contact, while Section 7 gives some implementation details. Section 8 presents numerical results and Section 9 concludes the paper.

\section{Problem setting and notations}

Let $\Omega \subset \mathbb{R}^{d}$ denote the reference configuration of a deformable solid in a space of dimension $d=2$ or 3 , where $\Omega$ may either be connected or consist of more than one connected components like for instance shown in Fig. 1. A deformed configuration of the considered solid can be defined through a transformation $\varphi$ which maps any point $X$ of the reference configuration to a new point $x$ :

$$
\begin{aligned}
\varphi: \Omega & \longrightarrow \mathbb{R}^{d} \\
X & \longmapsto x=\varphi(X),
\end{aligned}
$$


and is often written in terms of the displacement $u$ relatively to the reference configuration as:

$$
\varphi(X)=X+u(X) .
$$

In the deformed configuration $\Omega_{t}$, at time $t$, different portions of the boundary $\partial \Omega$ of $\Omega$ may come into contact and interact with each other. In order to express this interaction mathematically, it is convenient to consider part of $\partial \Omega$ as a slave (or contactor) surface $\Gamma^{S}$ and some other part as master (or target) surface $\Gamma^{M}$. Slave and master surfaces have to be defined in such a way that the corresponding surfaces $\Gamma_{t}^{S}$ and $\Gamma_{t}^{M}$ in the deformed configuration are likely to form a contact pair. A non-penetration condition between $\Gamma_{t}^{S}$ and $\Gamma_{t}^{M}$ can be expressed with the help of quantities defined in Table 1 and illustrated in Fig. 1. Recall that surface points $X, Y, x$ and $y$ are of dimension $d$ as well as the corresponding normal vectors $N_{X}, N_{Y}, n_{x}$ and $n_{y}$, while the deformation gradient $\mathbf{F}$, the identity matrix $\mathbf{I}$ and the projection operator $\mathbf{T}_{n}$ are $d \times d$ matrices.

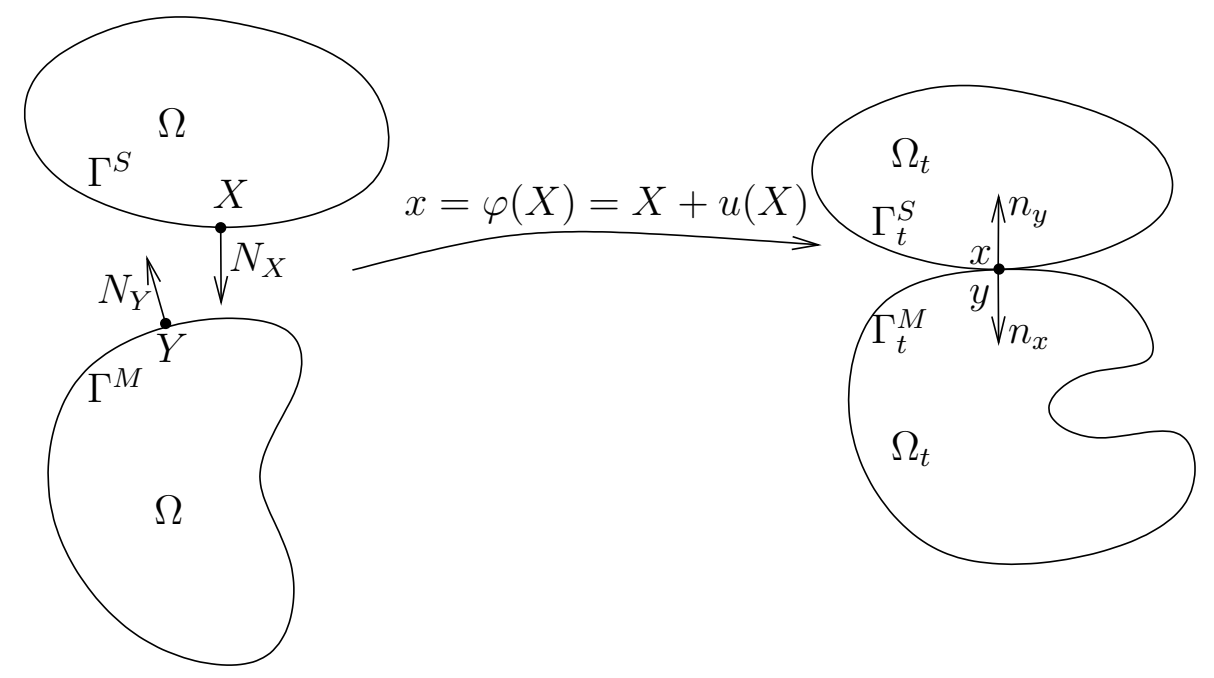

Figure 1: Contact interface quantities in reference and deformed configurations.

Deformation of the solid can be considered either in equilibrium or as part of a quasi-static evolution. Since the choice of a constitutive law is not central in the description of the proposed contact approximation, we will simply denote the global potential energy by $J(\varphi)$, without going into details about the different possible terms that may constitute this energy. As an example, if simple equilibrium under a gravity force is considered, the potential energy can have the form:

$$
J(\varphi)=\int_{\Omega} W(\varphi(X)) d X-\int_{\Omega} \rho \mathbf{g} \varphi(X) d X,
$$

where $W$ is the potential of a hyper-elastic constitutive law, $\rho$ is the density in the reference configuration and $\mathbf{g}$ is the gravity acceleration vector. Of course, additional terms such as boundary loads, can be considered as well.

Dirichlet conditions can also be prescribed, for instance, by constraining the displacements as well as the test functions of the weak formulation accordingly. For the sake of simplicity, the treatment of Dirichlet conditions will be omitted in the following.

Moreover, some special notation will help to simplify the mathematical presentation. The directional derivative of a quantity $A$ with respect to the deformation $\varphi$, at a point $x$ of the 
Table 1: Basic definitions.

\begin{tabular}{ll}
\multicolumn{1}{c}{ Expression } & \multicolumn{1}{c}{ Description } \\
\hline$\Omega$ & Reference configuration \\
$\Gamma^{S} \subset \partial \Omega$ & Slave (or contactor) surface \\
$\Gamma^{M} \subset \partial \Omega$ & Master (or target) surface \\
\hline$\Omega_{t}=\varphi(\Omega)$ & Deformed configuration (at time $t$ ) \\
$\Gamma_{t}^{S}=\varphi\left(\Gamma^{S}\right)$ & Slave surface in the deformed configuration \\
$\Gamma_{t}^{M}=\varphi\left(\Gamma^{M}\right)$ & Master surface in the deformed configuration \\
\hline$X \in \Gamma^{S}$ & Generic point on surface $\Gamma^{S}$ \\
$x=\varphi(X)$ & Point on surface $\Gamma_{t}^{S}$ corresponding to $X$ \\
$y$ & Point on surface $\Gamma_{t}^{M}$ that is mapped to \\
$Y: y=\varphi(Y)$ & point $x$ of surface $\Gamma_{t}^{S}$ \\
\hline $\mathbf{F}_{X}=\nabla \varphi(X)$ & Point on surface $\Gamma^{M}$ corresponding to $y$ \\
$\mathbf{F}_{Y}=\nabla \varphi(Y)$ & Deformation gradient at point $X$ \\
$N_{X}$ & Deformation gradient at point $Y$ \\
$N_{Y}$ & Unit outward normal vector to $\Gamma^{S}$ at $X$ \\
$n_{x}=\frac{\mathbf{F}_{X}^{-T} N_{X}}{\left\|\mathbf{F}_{X}^{-T} N_{X}\right\|}$ & Unit outward normal vector to $\Gamma^{M}$ at $Y$ \\
$n_{y}$ & Unit outward normal vector to $\Gamma_{t}^{S}$ at $x$ \\
\hline $\mathbf{T}_{n}=\mathbf{I}-n \otimes n$ & Unit outward normal vector to $\Gamma_{t}^{M}$ at $y$ \\
\hline & Projection operator onto the tangent \\
\hline & where $n$ can be e.g. $n_{x}$ or $n_{y}$ \\
\hline
\end{tabular}

deformed configuration and in direction $\delta u$ will be denoted by $\mathcal{D} A(x)[\delta u]$ or even by $\mathcal{D} A[\delta u]$ if the argument of the quantity $A$ is not ambiguous. The directional derivative is defined as:

$$
\mathcal{D} A(x)[\delta u]=\lim _{\varepsilon \rightarrow 0} \frac{A(x+\varepsilon \delta u)-A(x)}{\varepsilon}
$$

when this limit exists.

Some further notation to be used is the negative part operator $[\cdot]_{-}$, defined as:

$$
[x]_{-}=\left\{\begin{aligned}
-x & \text { if } x \leq 0 \\
0 & \text { if } x>0
\end{aligned}\right.
$$

\section{Ray-tracing instead of projection}

In the problem setting presented above, it is assumed that a point $x$ of the deformed slave surface can be mapped to a point $y$ of the deformed master surface, so that $y$ either coincides with $x$ or is expected to coincide with it as soon as contact occurs. Regarding this mapping, there are basically two possibilities, illustrated in Fig. 2. The most classic strategy is to define $y$ as the 
closest point projection of $x$ onto the deformed master surface $\Gamma_{t}^{M}$, like shown in Fig. 2(a). An alternative strategy, corresponding to Fig. 2(b), is to define $y$ as the closest intersection of the master surface $\Gamma_{t}^{M}$ with the line passing through point $x$ and having direction vector $n_{x}$. The latter strategy, which can be referred to as ray-tracing, will be used in the present paper for defining the mapping between slave and master surfaces and the corresponding gap function.

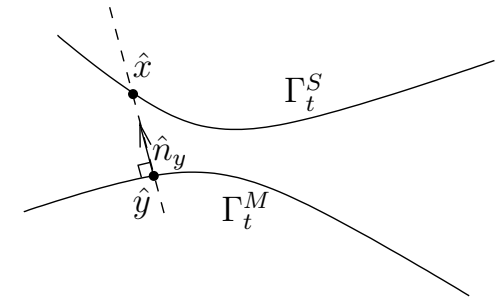

(a) Projection strategy

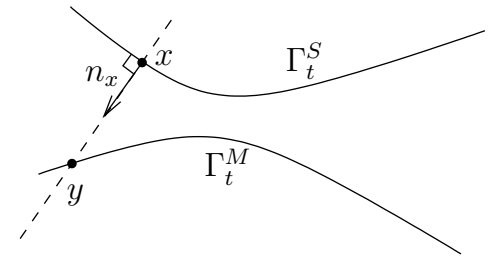

(b) Ray-tracing strategy

Figure 2: Comparison between projection and ray-tracing strategies.

The two strategies are in fact closely related since the orthogonality of $n_{x}$ to the slave surface implies that in the ray-tracing strategy, point $x$ is the projection of $y$ on the deformed slave surface $\Gamma_{t}^{S}$. For this reason, this strategy can also be characterized as an inverse projection. The main motivation for using it instead of the classical projection is for achieving a simpler expression for the weak formulation, due to the fact that the unit normal vector $n_{x}$ has a simpler derivative than $n_{y}$.

Contact kinematics are actually for both methods very similar. In order to make similarities and differences easier to recognize, the most important equations for both ray-tracing and projection are presented in parallel, with quantities referring to the projection method identified by an additional hat symbol.

Gap functions corresponding to ray-tracing and projection with respect to a point $x(X)$ or $\hat{x}(\hat{X})$ are respectively defined by:

$$
\begin{aligned}
& g=n_{x} \cdot(y-x), \\
& \hat{g}=\hat{n}_{y} \cdot(\hat{x}-\hat{y}),
\end{aligned}
$$

with these scalar expressions being based on the corresponding vector relations:

$$
\begin{aligned}
& y=x+g n_{x}, \\
& \hat{y}=\hat{x}-\hat{g} \hat{n}_{y},
\end{aligned}
$$

where $g$ and $\hat{g}$ are determined by the additional condition that $y$ or $\hat{y}$ lie on the deformed surface $\Gamma_{t}^{M}$.

For obtaining and linearizing a weak formulation representing the non-penetration condition, not only the definition of a gap function is required, but also the directional derivatives of all quantities involved in Eqs. (3) and (4) with respect to the current deformation $\varphi$ in a virtual direction $\delta u$. With $X$ or $\hat{X}$ considered as the independent variable, the directional derivatives of $x$ or $\hat{x}$ and $n_{x}$ are straightforward to determine, whether corresponding quantities at point $y$ are difficult to evaluate. This is because $y$ depends on both deformation $\varphi$ and coordinate $Y$, with the latter depending on $\varphi$ itself and having its own directional derivative $\mathcal{D} Y[\delta u]$. As a consequence, the directional derivatives of $y$ and $\hat{y}$ with respect to $\varphi$ can be written as:

$$
\mathcal{D} y[\delta u]=\delta u(Y)+\mathbf{F}_{Y} \mathcal{D} Y[\delta u]
$$




$$
\mathcal{D} \hat{y}[\delta u]=\delta u(\hat{Y})+\hat{\mathbf{F}}_{Y} \mathcal{D} \hat{Y}[\delta u],
$$

with $\mathcal{D} Y[\delta u]$ and $\mathcal{D} \hat{Y}[\delta u]$ being tangential to $\Gamma^{M}$, so that $\mathbf{F}_{Y} \mathcal{D} Y[\delta u]$ and $\hat{\mathbf{F}}_{Y} \mathcal{D} \hat{Y}[\delta u]$ are also tangential to the deformed surface $\Gamma_{t}^{M}$, which means:

$$
n_{y} \cdot \mathbf{F}_{Y} \mathcal{D} Y[\delta u]=\hat{n}_{y} \cdot \hat{\mathbf{F}}_{Y} \mathcal{D} \hat{Y}[\delta u]=0 .
$$

Alternative expressions about the directional derivatives of $y$ and $\hat{y}$ can be obtained from Eqs. (3) and (4) as:

$$
\begin{aligned}
& \mathcal{D} y[\delta u]=\delta u(X)+\mathcal{D} g[\delta u] n_{x}+g \mathcal{D} n_{x}[\delta u], \\
& \mathcal{D} \hat{y}[\delta u]=\delta u(\hat{X})-\mathcal{D} \hat{g}[\delta u] \hat{n}_{y}-\hat{g} \mathcal{D} \hat{n}_{y}[\delta u]
\end{aligned}
$$

Assuming sufficient regularity, the directional derivatives of the gap functions can be written after combining Eqs. (5) and (8) or Eqs. (6) and (9), multiplying respectively by $n_{y}$ or $\hat{n}_{y}$ and exploiting Eq. (7), as:

$$
\begin{gathered}
\mathcal{D} g[\delta u]=-\frac{n_{y}}{n_{x} \cdot n_{y}} \cdot\left(\delta u(X)-\delta u(Y)+g \mathcal{D} n_{x}[\delta u]\right), \\
\mathcal{D} \hat{g}[\delta u]=\hat{n}_{y} \cdot(\delta u(\hat{X})-\delta u(\hat{Y})) .
\end{gathered}
$$

The simpler expression in the case of projection is due to the fact that $\hat{n}_{y} \cdot \mathcal{D} \hat{n}_{y}[\delta u]=0$, but no similar relation can be utilized in case of ray-tracing. The directional derivative of the unit normal vector $n_{x}$ in Eq. (10) is given by:

$$
\mathcal{D} n_{x}[\delta u]=-\mathbf{T}_{n_{x}} \mathbf{F}_{X}^{-T} \nabla \delta u^{T}(X) n_{x} .
$$

At this point, despite the more complex expression obtained for $\mathcal{D} g[\delta u]$, the basic kinematic analysis of ray-tracing can be considered as completed. Substituting Eqs. (10) and (12) into Eq. (8) permits evaluation of $\mathcal{D} y[\delta u]$ while Eq. (5) can be used in a further step for evaluating $\mathcal{D} Y[\overline{\delta u}]$ as:

$$
\mathcal{D} Y[\delta u]=\mathbf{F}_{Y}^{-1}\left(\mathbf{I}-\frac{n_{x} \otimes n_{y}}{n_{x} \cdot n_{y}}\right)\left(\delta u(X)-\delta u(Y)-g \mathbf{F}_{X}^{-T} \nabla \delta u^{T}(X) n_{x}\right) .
$$

In the case of the projection strategy, Eq. (9) cannot be evaluated yet, since there is no closed form expression for $\mathcal{D} \hat{n}_{y}[\delta u]$ similar to Eq. (12). Apart from a term similar to Eq. $[12), \mathcal{D} \hat{n}_{y}[\delta u]$ involves $\mathcal{D} \hat{Y}[\delta u]$ and the curvature of the deformed master surface $\Gamma_{t}^{M}$ at point $\hat{y}$. With such an expression and combining Eqs. (6) and (9), it is possible to determine $\mathcal{D} \hat{Y}[\delta u]$ and consequently also $\mathcal{D} \hat{y}[\delta u]$ as described in detail, for instance, in [18, 30, 16].

From a computational viewpoint, ray-tracing allows a more efficient algorithm implementation than projection. The intersection equation for determining point $Y$ in ray-tracing is:

$$
(\varphi(Y)-\varphi(X)) \cdot t_{i}(X)=0
$$

while the projection equation for specifying point $\hat{Y}$ is:

$$
(\varphi(\hat{Y})-\varphi(\hat{X})) \cdot \hat{t}_{i}(\hat{Y})=0
$$

where vectors $t_{i}$ and $\hat{t}_{i}$, for $i=1, \ldots d-1$, form orthonormal bases of the planes tangent to $n_{x}$ and $\hat{n}_{y}$ respectively. After expressing $Y$ through $d-1$ coordinates on an element face of the 
discretized body, applying Newton's method for solving Eq. (14) is straightforward and efficient since its tangent system only involves $\mathbf{F}_{Y}$. The tangent system of Eq. (15) additionally involves the nonlinearity between the tangent basis vectors and the unknown $\hat{Y}$.

Moreover, there are generally less special cases to treat when dealing with ray-tracing rather than projection. The probability to come across a non regular point, like a corner of the geometry or simply an element boundary, is negligible for the ray-tracing strategy while it is very frequent for the projection. Fig. 3(a), illustrates how the projection from a significant portion of the slave surface can be somehow attracted by convex non-regular points, complicating the definition of $\hat{n}_{y}$. The probability that a ray-traced point $y$ in Fig. 3(b) falls on a non-regular point is negligible.

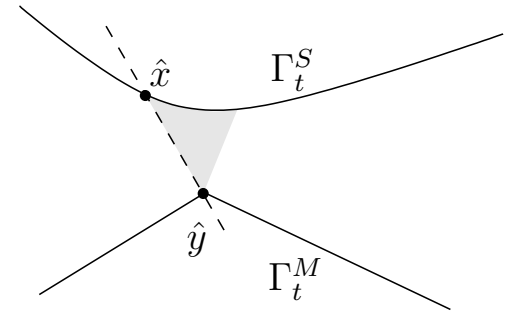

(a) Projection strategy

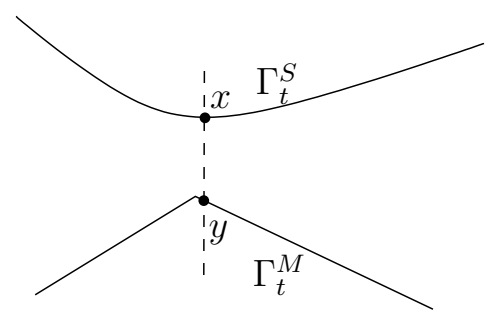

(b) Ray-tracing strategy

Figure 3: $\quad$ Set of slave points $\hat{x}$ projected onto a convex non-regular point $\hat{y}(a)$. Negligible probability of a ray-traced point y falling on a non-regular point (b).

\section{A weak formulation for frictionless contact}

In this section, a weak formulation for frictionless contact is obtained, which will be extended to frictional contact in Section 5 .

As described above, ray-tracing can be used for mapping a point $x=\varphi(X)$ on $\Gamma_{t}^{S}$ to a point $y$ on $\Gamma_{t}^{M}$. Let us then denote by $\Gamma_{c}^{S} \subset \Gamma^{S}$ the set of points $X$ in the reference configuration, for which such mapping exists. Force equilibrium of the considered elastic body, including the possibility of frictionless contact between master and slave surfaces, can be represented by the saddle point of the following Lagrangian:

$$
\mathscr{L}\left(\varphi, \lambda_{N}\right)=J(\varphi)+\int_{\Gamma_{c}^{S}} \lambda_{N}(X) g(X) d \Gamma,
$$

under the constraint $\lambda_{N} \leq 0$, where $\lambda_{N}: \Gamma^{S} \rightarrow \mathbb{R}$ is the Lagrange multiplier corresponding to the constraint $g \geq 0$. The multiplier $\lambda_{N}$ expresses force density in the reference configuration and combined with the unit normal $n_{x}$ in the deformed configuration, it expresses contact stress in the sense of the 1st Piola-Kirchhoff stress tensor.

Omitting argument $X$ from quantities $\lambda_{N}$ and $g$ for the sake of brevity, the optimality system of the Lagrangian function $\mathscr{L}$ within the set of admissible $\lambda_{N}$ values, is expressed in the following weak form:

$$
\left\{\begin{array}{l}
\mathcal{D} J(\varphi)[\delta u]+\int_{\Gamma_{c}^{S}} \lambda_{N} \mathcal{D} g[\delta u] d \Gamma=0 \quad \forall \delta u, \\
\int_{\Gamma_{c}^{S}}\left(\lambda_{N}-\delta \lambda_{N}\right) g d \Gamma \geq 0 \quad \forall \delta \lambda_{N} \leq 0,
\end{array}\right.
$$

still under the constraint $\lambda_{N} \leq 0$. 
In order to avoid the additional constraint on $\lambda_{N}$, the augmented Lagrangian function for inequality constrained systems can be considered. It was originally applied to contact mechanics problems by Alart and Curnier in [1] and it is adapted to our notation as:

$$
\mathscr{L}_{r}\left(\varphi, \lambda_{N}\right)=J(\varphi)+\frac{1}{2 r} \int_{\Gamma_{c}^{S}}\left[\lambda_{N}+r g\right]_{-}^{2}-\lambda_{N}^{2} d \Gamma
$$

where $r>0$ is the augmentation parameter.

The optimality system of $\mathscr{L}_{r}$ is the following:

$$
\left\{\begin{array}{l}
\mathcal{D} J(\varphi)[\delta u]-\int_{\Gamma_{c}^{S}}\left[\lambda_{N}+r g\right]_{-} \mathcal{D} g[\delta u] d \Gamma=0 \quad \forall \delta u \\
-\frac{1}{r} \int_{\Gamma_{c}^{S}}\left(\lambda_{N}+\left[\lambda_{N}+r g\right]_{-}\right) \delta \lambda_{N} d \Gamma=0 \quad \forall \delta \lambda_{N} .
\end{array}\right.
$$

Note that the use of $\mathscr{L}_{r}$ does not represent an approximation or a penalization of the contact condition. In the continuous setting and neglecting regularity issues, both the unconstrained Lagrangian function $\mathscr{L}_{r}$ and the Lagrangian function $\mathscr{L}$ under the constraint $\lambda_{N} \leq 0$, have the same saddle point. The advantage of using the augmented Lagrangian (18) is that System (19) does not involve inequality constraints. Additionally, the choice of expressing System (19) as a weak formulation both with respect to the deformation $\varphi$ and the multiplier $\lambda_{N}$, offers flexibility regarding its discretization. If one would alternatively choose to define $\mathscr{L}_{r}$ with respect to a nodal or element-wise weighted discrete version of the gap $g$, as it is very common, the second equation in System 19. would be replaced by an expression resembling:

$$
\int_{\Gamma_{c}^{S}} \lambda_{N} \delta \lambda_{N} d \Gamma+\left[\int_{\Gamma_{c}^{S}} \lambda_{N} \delta \lambda_{N} d \Gamma+r \int_{\Gamma_{c}^{S}} g \delta \lambda_{N} d \Gamma\right]_{-}=0,
$$

for a chosen set of test functions $\delta \lambda_{N}$ depending on the discretization. Eq. 200 is consistent with the second equation in System (19) only in the case of a nodal approximation, where $\delta \lambda_{N}$ are Dirac delta functions. In the case of mortar approximations, Eq. (20), apart from not representing accurately the underlying continuous equation, it also poses limitations to the choice of test functions $\delta \lambda_{N}$. These have to be chosen so that the two types of integrals appearing in Eq. (20) represent discrete multipliers and weighted gaps respectively.

Nevertheless, the first equation of System $(19)$ poses some significant difficulties. The master surface unit normal vector $n_{y}$, which appears in $\mathcal{D} g[\delta u]$ through Eq. $(10)$, is not even continuous with respect to $x=\varphi(X)$. As illustrated in Fig. 4(a), when a point of the slave surface slides across a corner or element boundary on the master surface, a jump in the normal vector $n_{y}$ may occur for both ray-tracing and projection strategies. Such a discontinuity is an obstacle for obtaining the tangent of the optimality system $\sqrt{19}$ in order to apply Newton's algorithm and can result to poor convergence. 


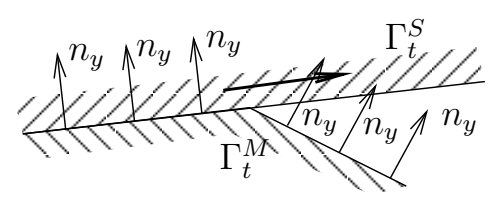

(a) Discontinuity of $n_{y}$

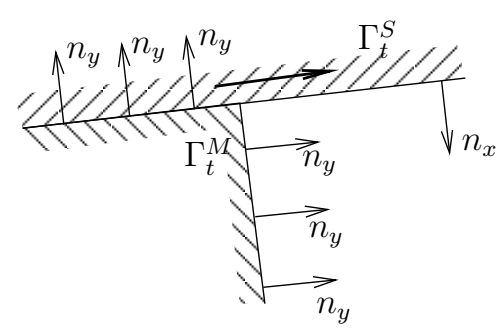

(b) No corresponding point $y$ found

Figure 4: Possible discontinuities of $n_{y}$ with respect to $x$.

In order to circumvent this difficulty, we remark that at any point of the slave surface, either contact occurs so that $n_{y}=-n_{x}$, or contact does not occur so that $\left[\lambda_{N}+r g\right]_{-}$vanishes. This means that, remaining consistent, the term $\frac{n_{y}}{n_{x} \cdot n_{y}}$ that would appear in the optimality system due to $\mathcal{D} g[\delta u]$ can be simply replaced by $n_{x}$. Remarking additionally that $n_{x} \cdot \mathcal{D} n_{x}[\delta u]=0$, substitution of Eq. (10) into Eq. (19) leads to the following simplified system:

$$
\left\{\begin{array}{l}
\mathcal{D} J(\varphi)[\delta u]-\int_{\Gamma_{c}^{S}}\left[\lambda_{N}+r g\right]_{-} n_{x} \cdot(\delta u(X)-\delta u(Y)) d \Gamma=0 \quad \forall \delta u, \\
-\frac{1}{r} \int_{\Gamma_{C}^{S}}\left(\lambda_{N}+\left[\lambda_{N}+r g\right]_{-}\right) \delta \lambda_{N} d \Gamma=0 \quad \forall \delta \lambda_{N},
\end{array}\right.
$$

with the actual advantage that it involves only the unit normal vector $n_{x}$, which is differentiable with respect to the deformation, see Eq. (12).

Optionally, the second line of System (21) can be exploited for replacing the term $\left[\lambda_{N}+r g\right]_{-}$ in the first line of System (21) with $-\lambda_{N}$, resulting to the following simpler but non-symmetric system:

$$
\left\{\begin{array}{l}
\mathcal{D} J(\varphi)[\delta u]-\int_{\Gamma_{c}^{S}} \lambda_{N} n_{x} \cdot(\delta u(X)-\delta u(Y)) d \Gamma=0 \quad \forall \delta u, \\
-\frac{1}{r} \int_{\Gamma_{c}^{S}}\left(\lambda_{N}+\left[\lambda_{N}+r g\right]_{-}\right) \delta \lambda_{N} d \Gamma=0 \quad \forall \delta \lambda_{N} .
\end{array}\right.
$$

Regarding the lack of symmetry in System (22), numerical tests in [25] showed that, at least under small deformations, non-symmetric formulations do not necessarily perform worse in terms of Newton's iterations than symmetric ones. Note also that action-reaction Newton's law is obviously satisfied in (22).

A possible discontinuity which can still affect the solution of System (22) is shown in Fig. 4(b). In that case, after a point $x$ of the slave surface passes the corner of the master surface, ray-tracing or projection will fail to find a corresponding point $y$ on the master surface. Hence, the considered point $X$ in the reference configuration is no longer within $\Gamma_{c}^{S}$. This remaining discontinuity in the system is difficult to avoid since the real contact stress is in this case indeed discontinuous.

As a conclusion, the obtained system 22 is relatively simple, avoids the great majority of pathological discontinuities with respect to the deformation and does not include inequality constraints. The price for this simplicity and regularity is a certain loss of symmetry. 


\section{A weak formulation for contact with Coulomb friction}

In the presence of friction, normal and tangential stresses in the contact interface are coupled through the sliding velocity vector. This means that friction normally applies to transient problems, although there are also static equilibrium cases that have a physical sense. In order to extend our contact formulation to Coulomb friction, a quasi-static process will be considered with $J(\varphi)$ still representing the total potential energy and $\mathrm{v}(X)$ denoting the relative velocity at a point $X$ in the contact interface.

The frame indifferent definition of $\mathrm{v}(X)$ described in [5], adapted to ray-tracing and the current notation reads:

$$
\mathrm{v}(X)=\dot{\varphi}(X)-\dot{\varphi}(Y)+g \dot{n}_{x}=\mathbf{F}_{Y} \dot{Y}-\dot{g} n_{x}
$$

with dotted quantities representing time derivatives. In this paper, time discretization is based on a backward Euler approximation of the first expression in Eq. (23) which reads:

$$
\mathrm{v}(X)=\frac{1}{\Delta t}\left(\varphi(X)-\varphi(Y)+g n_{x}\right)-\frac{1}{\Delta t}\left(\varphi_{0}(X)-\varphi_{0}(Y)+g n_{x 0}\right),
$$

where $\varphi_{0}$ and $n_{x 0}$ are respectively the deformation and the slave surface normal at the previous time step. Eq. (24) can be then simplified further, based on Eq. (3), as following:

$$
\mathrm{v}(X)=-\frac{1}{\Delta t}\left(\varphi_{0}(X)-\varphi_{0}(Y)+g n_{x 0}\right) .
$$

It should be underlined here that the mapping between points $X$ and $Y$ appearing in Eq. (25) corresponds to the current deformation $\varphi$ and not the deformation $\varphi_{0}$ at the previous time step.

Contact with Coulomb friction cannot be represented by the saddle point of a Lagrangian function similar to (18). This is possible only for Tresca friction, where there is no coupling between normal and tangential stresses in the contact interface. Among others, references [1, 25] obtain an unconstrained system corresponding to 22 for Coulomb friction by modifying appropriately the optimality system of a Lagrangian function representing Tresca friction. One result of this treatment is that impenetrability, Coulomb friction as well as the relevant complementarity conditions can be expressed through a non-smooth equality condition:

$$
\mathcal{C}(\lambda, g, \mathrm{v}, n)=0
$$

with $\mathcal{C}$ defined according to the notation followed in the current paper as:

$$
\mathcal{C}(\lambda, g, \mathrm{v}, n)=\lambda+[\lambda \cdot n+r g]_{-} n-P_{B\left(n, \mathscr{F}[\lambda \cdot n+r g]_{-}\right)}(\lambda-r \mathrm{v}),
$$

where $\mathscr{F}$ is the Coulomb friction coefficient and $\lambda: \Gamma_{c}^{S} \rightarrow \mathbb{R}^{d}$ is a contact traction similar to the 1st Piola-Kirchhoff stress tensor in continuum mechanics. More specifically, $\lambda$ represents force vectors evaluated in the deformed configuration divided by contact areas evaluated in the reference configuration. Unlike reference [25] where $P_{B(\tau)}$ represents a simple ball projection, here $P_{B(n, \tau)}$ is a projection on the ball of radius $\tau$ and at the same time onto the tangent plane defined by the normal $n$, i.e.:

$$
P_{B(n, \tau)}(q)= \begin{cases}\mathbf{T}_{n} q & \text { if }\left\|\mathbf{T}_{n} q\right\| \leq \tau, \\ \tau \frac{\mathbf{T}_{n} q}{\left\|\mathbf{T}_{n} q\right\|} & \text { otherwise. }\end{cases}
$$


Eqs. 26) and (27) form the basis for a series of fixed point strategies found in the literature, summarized for instance in [17. Nevertheless, here Eq. (26) will be enforced in an integral sense, equivalent to the approach presented in the previous section for frictionless contact. For this purpose, System 22 is extended to include Coulomb friction as following:

$$
\left\{\begin{array}{l}
\mathcal{D} J(\varphi)[\delta u]-\int_{\Gamma_{c}^{S}} \lambda \cdot(\delta u(X)-\delta u(Y)) d \Gamma=0 \quad \forall \delta u, \\
-\frac{1}{r} \int_{\Gamma_{c}^{S}} \mathcal{C}\left(\lambda, g, \mathrm{v}, n_{x}\right) \cdot \delta \lambda d \Gamma=0 \quad \forall \delta \lambda .
\end{array}\right.
$$

Note that the non-smooth complementarity function $\mathcal{C}$, defined in (27), expresses Coulomb's law on $\lambda$ which is the contact force density in the reference configuration. The real contact force density in the deformed configuration is:

$$
\bar{\lambda}=\frac{\lambda}{\left\|\mathbf{F}_{X}^{-T} N_{X}\right\| \operatorname{det}\left(\mathbf{F}_{X}\right)} .
$$

The fact that Coulomb's law is not sensitive to the absolute magnitude of the tangential and normal contact forces allows to express it in the reference configuration. This would not be the case for more elaborate laws, for instance, when the friction threshold depends nonlinearly on the contact pressure.

\section{Finite element approximation and tangent system}

Reference [25], although restricted to small deformations, it provides a review on different approximations of the frictional contact condition, which are still applicable to the large deformations setting. One option for solving System (29) numerically, is a nodal approximation of the contact condition in combination with a Lagrange finite element discretization of the force equilibrium equation. However, a nodal contact condition involves the unit normal vector $n_{x}$ at finite element nodes. Except for conformal $C^{1}$ continuous finite elements, the surface unit normal vector is not well defined at nodes on the boundary between elements, obliging to work with averaged unit normals, like for instance in [24]. Although this is not a huge difficulty, the present paper focuses on an integral approximation of contact and friction conditions in 29 , so that, at the discrete level, the normal vector $n_{x}$ is evaluated at quadrature points on the slave surface, where it is well defined.

Since System (29) is an unconstrained problem, a standard Galerkin procedure can be applied by choosing two finite element spaces for the displacements and the contact stress fields. In particular, it is not necessary to further specify in what sense the normal contact stress is non-positive or in what sense the Coulomb friction law is fulfilled since it is already taken into account. Of course, the chosen finite element spaces have to fulfill a discrete inf-sup (also known as Babuska-Brezzi) stability condition, as explained for instance in [7. For frictionless contact in two dimensions, it has been demonstrated numerically in [7] and theoretically in [3], that a 
continuous piecewise linear approximation of the contact stress combined with continuous piecewise linear or quadratic approximations of the displacements results to stable schemes (cases L1/L1 and L2/L1 respectively). An independent proof for the case of linear approximations (L1/L1) can be found also in [4]. As demonstrated in [14, combination of continuous piecewise quadratic approximations for both the contact stress and the displacements (case L2/L2) also satisfies the considered inf-sup condition. In the two-dimensional numerical examples included in the present work, all three aforementioned discretization schemes are considered, while in the three-dimensional example only the combination L2/L1 is used.

Let $V^{h} \subset H^{1}\left(\Omega ; \mathbb{R}^{d}\right)$ and $W^{h} \subset L^{2}\left(\Gamma^{S} ; \mathbb{R}^{d}\right)$ be two finite element spaces corresponding to the displacement and the contact multiplier fields respectively. It is assumed that $V^{h}$ accounts for any possible Dirichlet condition and that the pair $V^{h}, W^{h}$ satisfies the aforementioned inf-sup condition. Then, the finite element approximation of System 29 reads:

$$
\left\{\begin{array}{l}
\mathcal{D} J\left(\varphi^{h}\right)\left[\delta u^{h}\right]-\int_{\Gamma_{c}^{S}} \lambda^{h} \cdot\left(\delta u^{h}(X)-\delta u^{h}(Y)\right) d \Gamma=0 \quad \forall \delta u^{h} \in V^{h}, \\
-\frac{1}{r} \int_{\Gamma_{c}^{S}} \mathcal{C}\left(\lambda^{h}, g, \mathrm{v}, n_{x}\right) \cdot \delta \lambda^{h} d \Gamma=0 \quad \forall \delta \lambda^{h} \in W^{h} .
\end{array}\right.
$$

Note that, excepting the case of Fig. 4(b), System (31) is Lipschitz-continuous with respect to the pair $\varphi^{h}, \lambda^{h}$ and piecewise $C^{1}$ continuous. This means that it is sufficiently regular to be solved with a generalized Newton method. The tangent system of (31), which is necessary for the use of Newton's algorithm, is provided below. Each Newton step consists in finding $\Delta \lambda^{h} \in W^{h}$ and $\Delta u^{h} \in V^{h}$ as a solution to:

$$
\left\{\begin{array}{rlr}
\mathcal{D}^{2} J\left(\varphi^{h}\right)\left[\delta u^{h}, \Delta u^{h}\right] & \\
-\int_{\Gamma_{c}^{S}} \Delta \lambda^{h} \cdot\left(\delta u^{h}(X)-\delta u^{h}(Y)\right)-\lambda^{h} \cdot \nabla \delta u^{h}(Y) \mathcal{D} Y\left[\Delta u^{h}\right] d \Gamma & \\
=-\mathcal{D} J\left(\varphi^{h}\right)\left[\delta u^{h}\right]+\int_{\Gamma_{c}^{S}} \lambda^{h} \cdot\left(\delta u^{h}(X)-\delta u^{h}(Y)\right) d \Gamma & \forall \delta u^{h} \in V^{h}, \\
-\frac{1}{r} \int_{\Gamma_{c}^{S}}\left[\partial_{\lambda} \mathcal{C} \Delta \lambda^{h}+\partial_{g} \mathcal{C} \mathcal{D} g\left[\Delta u^{h}\right]+\partial_{\mathrm{v}} \mathcal{C} \mathcal{D v}\left[\Delta u^{h}\right]+\partial_{n} \mathcal{C} \mathcal{D} n_{x}\left[\Delta u^{h}\right]\right] \cdot \delta \lambda^{h} d \Gamma \\
=\frac{1}{r} \int_{\Gamma_{c}^{S}} \mathcal{C} \cdot \delta \lambda^{h} d \Gamma & \forall \delta \lambda^{h} \in W^{h},
\end{array}\right.
$$

where $\mathcal{D}^{2} J\left(\varphi^{h}\right)\left[\delta u^{h}, \Delta u^{h}\right]$ is the second directional derivative of $J\left(\varphi^{h}\right)$, while derivatives $\mathcal{D} g\left[\Delta u^{h}\right]$, $\mathcal{D} n_{x}\left[\Delta u^{h}\right]$ and $\mathcal{D} y\left[\Delta u^{h}\right]$ are given respectively by Eqs. (10), (12) and (8). Based on Eq. (24), the derivative $\operatorname{Dv}\left[\Delta u^{h}\right]$ can be evaluated according to:

$$
\mathcal{D} \mathrm{v}[\delta u]=\frac{1}{\Delta t}\left(\delta u(X)-\delta u(Y)-\left(\mathbf{F}_{Y}-\mathbf{F}_{Y 0}\right) \mathcal{D} Y+\mathcal{D} g\left(n_{x}-n_{x 0}\right)+g \mathcal{D} n_{x}\right),
$$

or alternatively based on Eq. (25) as:

$$
\mathcal{D} \mathrm{v}[\delta u]=\frac{1}{\Delta t}\left(\mathbf{F}_{Y 0} \mathcal{D} Y-\mathcal{D} g n_{x 0}\right)
$$

where $\mathbf{F}_{Y 0}$ is the deformation gradient at the previous time step, evaluated at point $Y$. Finally, all partial derivatives $\partial_{\lambda} \mathcal{C}, \partial_{g} \mathcal{C}, \partial_{\mathrm{v}} \mathcal{C}$ and $\partial_{n} \mathcal{C}$ of function $\mathcal{C}$ are provided in Appendix A 


\section{A few implementations details}

The here proposed approximation has been implemented in the open-source finite element library GetFEM++, [22]. In this implementation System (32) can be solved iteratively in the context of Newton's algorithm for arbitrary finite element spaces $V^{h}$ and $W^{h}$. Nevertheless, the numerical examples included in the present paper are limited to different combinations of linear and quadratic Lagrange finite elements.

With regard to numerical integration, although System (32) involves only surface integrals defined on the undeformed slave surface, these contain integrands referring to both the slave and master surfaces. As a consequence, the generally non-matching meshes between the discretized surfaces in contact pose a significant difficulty for achieving accurate integration. Moreover, the boundary between the smooth subregions of the non-smooth function $\mathcal{C}$ is in general a priori unknown so that exact evaluation of the corresponding integrals is not possible. For this reason, an approximate integration has to be considered. All numerical examples presented in the current paper are based on Legendre-Gauss quadrature on the faces of the mesh elements belonging to the slave surface. Tests with an increasing number of quadrature points showed that despite the low regularity of the integrated functions, relatively few quadrature points are sufficient for achieving reasonable accuracy. In most cases, four quadrature points per edge in two-dimensional problems and sixteen quadrature points per element face in three-dimensional problems provide satisfactory results.

Contact detection is performed on each quadrature point independently. As a consequence, different quadrature points on a single element face of the slave surface, can be associated to points on different element faces of the master surface. A rather simple heuristic method based on R-tree organized influence boxes is used for finding candidate master element faces in front of a particular quadrature point in computational time of logarithmic complexity. For curved element faces, Newton's algorithm is used for performing ray-tracing according to Eq. (14) and the ray-traced point $Y$ is specified in terms of element local coordinates. In order to reduce the number of candidate target faces, a release distance criterion is applied so that only contact pairs which are likely to occur within the current load step, are considered. Further details about the applied heuristic criteria for contact detection are provided in the user documentation of GetFEM++, 22.

A practical issue related to the proposed method concerns the treatment of the subregion $\Gamma^{S} \backslash \Gamma_{c}^{S}$ of a predefined slave surface $\Gamma^{S}$, where ray-tracing will not yield a corresponding master surface point, for instance due to the predefined release distance been exceeded. Without any special treatment, the contact multiplier within this subregion would become indefinite. In order to avoid this situation the term

$$
-\frac{1}{r} \int_{\Gamma^{S} \backslash \Gamma_{c}^{S}} \lambda^{h} \cdot \delta \lambda^{h} d \Gamma
$$

is added to the second line of System (31) for all quadrature points lying in the aforementioned subregion $\Gamma^{S} \backslash \Gamma_{c}^{S}$.

Finally, it should be noted that the system of linear equations $(32)$ to be solved in each Newton's iteration, represents a non-symmetric system with no special structure that would, for instance, allow an easy elimination of Lagrange multipliers or the usage of specialized efficient iterative solvers. Nevertheless, the proposed method is applicable to problems of considerable size by employing a parallelized direct solver, which can efficiently deal with non-symmetric systems and systems representing saddle-point problems. Our implementation relies on the sparse direct linear solver MUMPS [2]. 


\section{Numerical examples and discussion}

Particularities and performance characteristics of the proposed method are illustrated in this section through numerical examples. As a first example, simulation of a two-dimensional Hertzian contact problem shows the capability of the proposed integral approximation to capture a contact pressure profile of known form. Two further two-dimensional examples are classical problems found in the large sliding contact literature and aim at giving an impression about the convergence performance of the proposed method. Finally, simulation of contact between two hollow cylindrical tubes, including self-contact, is presented in order to evaluate the performance of the method in three dimensions.

All four examples are based on a neo-Hookean material law according to the following strain energy density function:

$$
W(\varphi)=\frac{G}{2}\left(i_{1}-3\right)+\frac{\Lambda}{4}\left(i_{3}-1\right)-\left(\frac{G}{2}+\frac{\Lambda}{4}\right) \ln \left(i_{3}\right)
$$

with $i_{1}$ and $i_{3}$ respectively representing the first and the third invariants of the right CauchyGreen tensor, defined according to the current notation as $\mathbf{F}^{T} \mathbf{F}$. Lamé's elasticity parameters $\Lambda$ and $G$ can be calculated from the corresponding Young's modulus $E$ and Poisson's ratio $\nu$ values, provided in each numerical example.

\subsection{Hertzian contact}

The most important characteristics of enforcing contact complementarity conditions in an integral sense can be explained on the basis of a Hertzian contact problem. The main particularity, compared to nodal or element-wise enforcements of contact conditions, actually concerns the boundary of the contact region. Finite element faces which are intersected by the boundary of the contact region contribute to both contact and non-contact terms quadrature-point-wise. Considering frictionless contact for simplicity, quadrature points in the non-contact region contribute with the term:

$$
-\frac{1}{r} \lambda_{N} \delta \lambda_{N}
$$

to the second integral of System (22), while quadrature points within the contact region contribute to the same integral with the term:

$$
g \delta \lambda_{N} .
$$

The first of these two terms enforces a vanishing contact pressure outside the contact region, while the second one enforces a vanishing gap within the contact region. One consequence of this observation is that the actual solution depends on the augmentation parameter $r$ in the sense that lower values of $r$ will practically favor the non-negativity of the contact pressure outside the contact region while higher values of $r$ will favor the non-penetration condition, when both conditions cannot be fulfilled at the same time due to the limited accuracy of the chosen finite element approximation. The fact that classical finite element approximations of the displacement and contact pressure fields cannot fulfill complementarity conditions with respect to any arbitrary contact region exactly, is a fundamental limitation which is not specific to the here employed method.

Fig. 5 shows calculated pressure profiles for frictionless contact between a half-disc of radius equal to $1 \mathrm{~mm}$ and a flat rigid obstacle. The half-disc material parameters corresponding to Eq. (35) are chosen so that under infinitesimal strains, linearized elasticity with $E=10^{5} \mathrm{MPa}$ 
and $\nu=0.3$ is approximated. The half-disc top side is clamped and lowered vertically in steps of $0.01 \mathrm{~mm}$. The diagrams shown in the first and second row of Fig. 5 correspond to the 16th and 18th load step respectively. The knots shown in the diagrams correspond to nodes of the finite element approximation of the displacements field while the vertical red arrows correspond to values of the contact multiplier field at quadrature points. Specifically referring to Fig. 5 both displacements and contact multiplier fields are approximated with linear Lagrange elements and four quadrature points per element face. It should be noted that the illustrated contact pressure values correspond to the corrected multiplier $\bar{\lambda}$ defined in Eq. (30). The dashed blue line represents the analytically calculated Hertzian pressure profile for the corresponding normal load obtained in the simulation. It should be noted that the studied problem is not strictly a Hertzian contact due to the high load resulting to a contact width of considerable size compared to the disc radius. However, numerical experiments showed that the considered material law (35) preserves the validity of the Hertzian solution in the finite strain regime quite accurately.
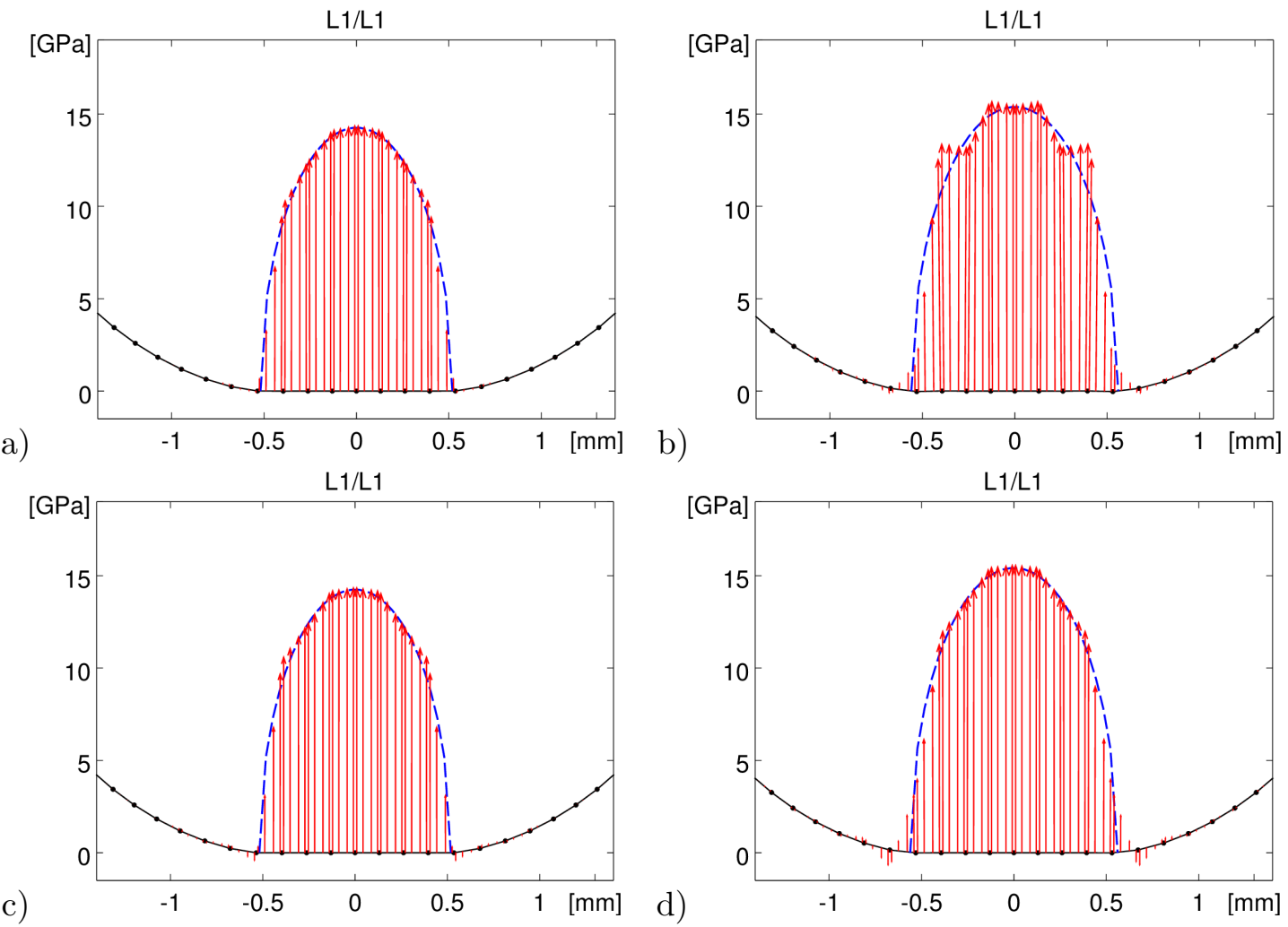

Figure 5: $\quad$ Calculated contact pressure profiles for vertical loads of $13.8 \mathrm{kN}$ in diagrams (a),(c) and 16.2 $\mathrm{kN}$ in diagrams (b),(d), obtained with $r=E /(1 \mathrm{~mm})$ for diagrams (a),(b) and $r=E /(0.01 \mathrm{~mm})$ for diagrams $(c),(d)$.

A first observation regarding Fig. 5, concerns the approximation error being highly dependent on the load step. A comparison between diagrams a) and b) shows that a linear finite element approximation of the contact pressure field can capture the expected pressure profile in the 16th load step quite accurately while it results to a significantly worse approximation in the 18th load step. Actually the observed approximation error depends on the alignment between discontinuities of the real field to be approximated and the finite element nodes. 
A further observation concerns the anticipated impact of the augmentation parameter $r$ on the numerical results, confirmed by comparing diagrams c) and d) with a) and b) respectively. It is clear that the sensitivity of the solution on $r$ increases with increasing incompatibility between the considered finite element approximation and the approximated solution, like for instance in cases b) and d).

We remark here that by enforcing contact complementarity conditions in an integral sense, it is possible for the contact multiplier to become negative not only at individual quadrature points but also at nodal values of its finite element approximation, as indicated for instance in Fig. 5 d).

a)

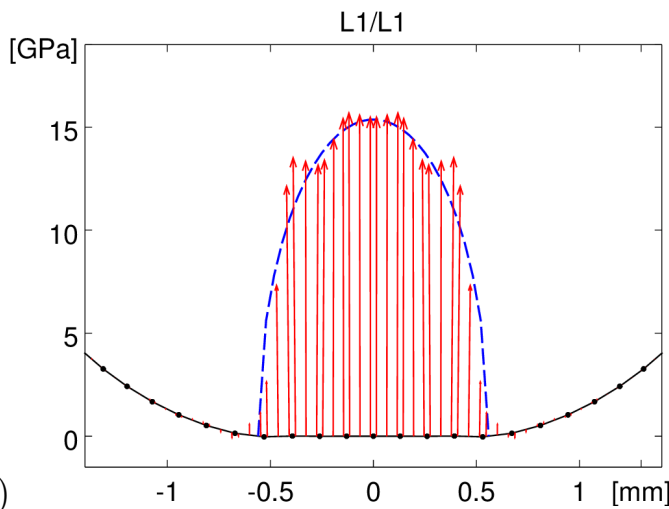

c)
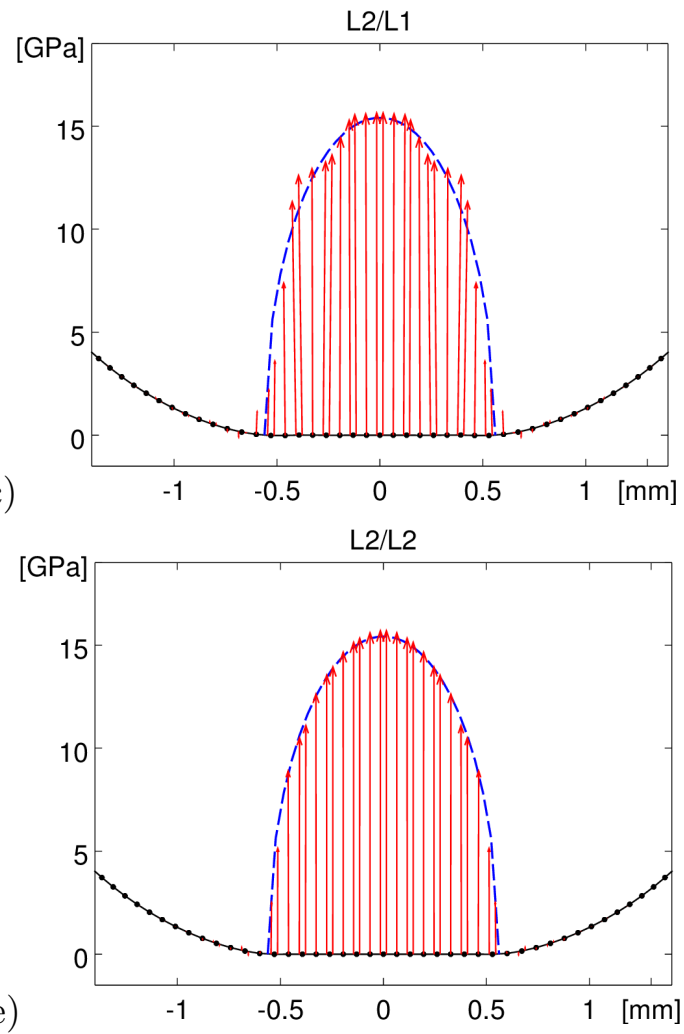

b)

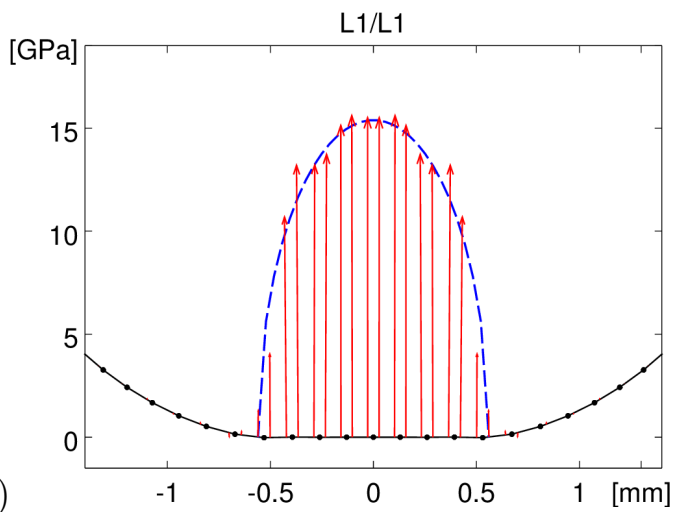

d)
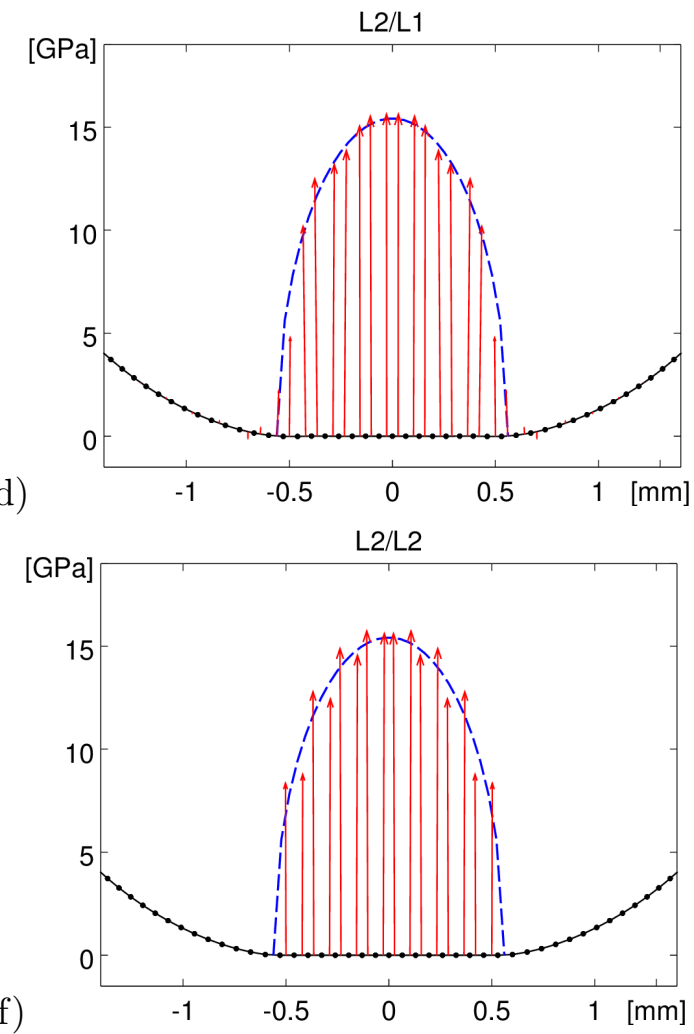

Figure 6: Calculated contact pressure profiles for vertical load of $16.2 \mathrm{kN}$, obtained with three and two quadrature points per element face for the left and the right column respectively and for different combinations of linear and quadratic finite element approximations. 
Fig. 6] shows results corresponding to the 18th load step obtained with three quadrature points per element face for the diagrams in the left column and two quadrature points for the ones in the right column. All results shown in this figure are obtained with the augmentation parameter $r$ equal to Young's Modulus $E$ divided by a characteristic length of $1 \mathrm{~mm}$. Diagrams in the first row correspond to linear approximations for both displacements and contact multiplier fields while the results shown in the middle row refer to a quadratic approximation of the displacements field only and the diagrams in the last row refer to a quadratic approximation of both fields. Some aspects revealed by Fig. 6 are the following:

- The approximation of the pressure profile improves slightly with quadratic finite elements for the displacements, but improves more significantly when quadratic finite elements are used also for the contact multiplier.

- As an exception to the previous observation, quadratic approximation of both fields fails to provide a correct result in the case of only two quadrature points, due to poor numerical integration.

- For linear approximation of the contact multiplier field, two quadrature points per element face appear to be sufficient for numerical integration.

Concluding this numerical example, it appears that the proposed integral enforcement of contact conditions, combined with quadratic finite elements for both the displacements and contact multiplier fields and using three quadrature points per element faces appears to be very accurate. Although, for the sake of space, only one load step regarding this preferred setting is shown in Fig. 6 e), it should be noted that the quality of the approximation is very insensitive to the alignment between the approximated pressure profile and the finite element nodes. Actually, the result shown in Fig. 6 e) for the 18th load step represents one of the worst cases among all calculated load steps.

\subsection{Loaded elastic half-ring}

The second numerical example is one of the contact problems presented in [9] and [28]. It involves an elastic half-ring consisting of two layers of different elastic properties, which is loaded against an elastic rectangular block. As in reference [28], both parts are assumed to exhibit neo-Hookean material behavior with Poisson's ratio equal to 0.3 and initial Young's moduli equal to $10^{5}$ and $10^{3} \mathrm{MPa}$ for the inner and outer ring layers respectively and $300 \mathrm{MPa}$ for the block. The inner diameter of the half-ring is equal to $90 \mathrm{~mm}$, while the thickness of each ring layer is equal to $5 \mathrm{~mm}$. The block is $260 \mathrm{~mm}$ long and $50 \mathrm{~mm}$ high and the initial gap between the ring and the block is equal to $20 \mathrm{~mm}$.

Fig. 7 shows the initial geometry and four deformed configurations at different simulation steps, obtained without and with friction. The coarsest mesh utilized in the calculations consists of 64 elements along the ring circumference and 1 element across each ring layer thickness, while the block is discretized through 52 by 10 quadrilateral elements in length and height directions respectively. During the conducted simulation, the rectangular block is fixed at its bottom edge, while the ends of the half-ring are horizontally fixed and vertically displaced from $-20 \mathrm{~mm}$ to $-90 \mathrm{~mm}$ in steps of $0.5 \mathrm{~mm}$.

The results shown in Fig. 7 were obtained using 9-node quadrilateral second order finite elements for approximating the displacements field and a linear approximation for the Lagrange

multiplier representing the contact stress. The outer ring edge was defined as the slave surface 
with eight quadrature points per contact element and the top block edge was defined as the master surface. In accordance with the conclusions of the previous example, an augmentation parameter value of $300 \mathrm{MPa} / \mathrm{mm}$ was chosen, which corresponds to the Young's modulus of the most compliant component in the system and a characteristic length of $1 \mathrm{~mm}$.
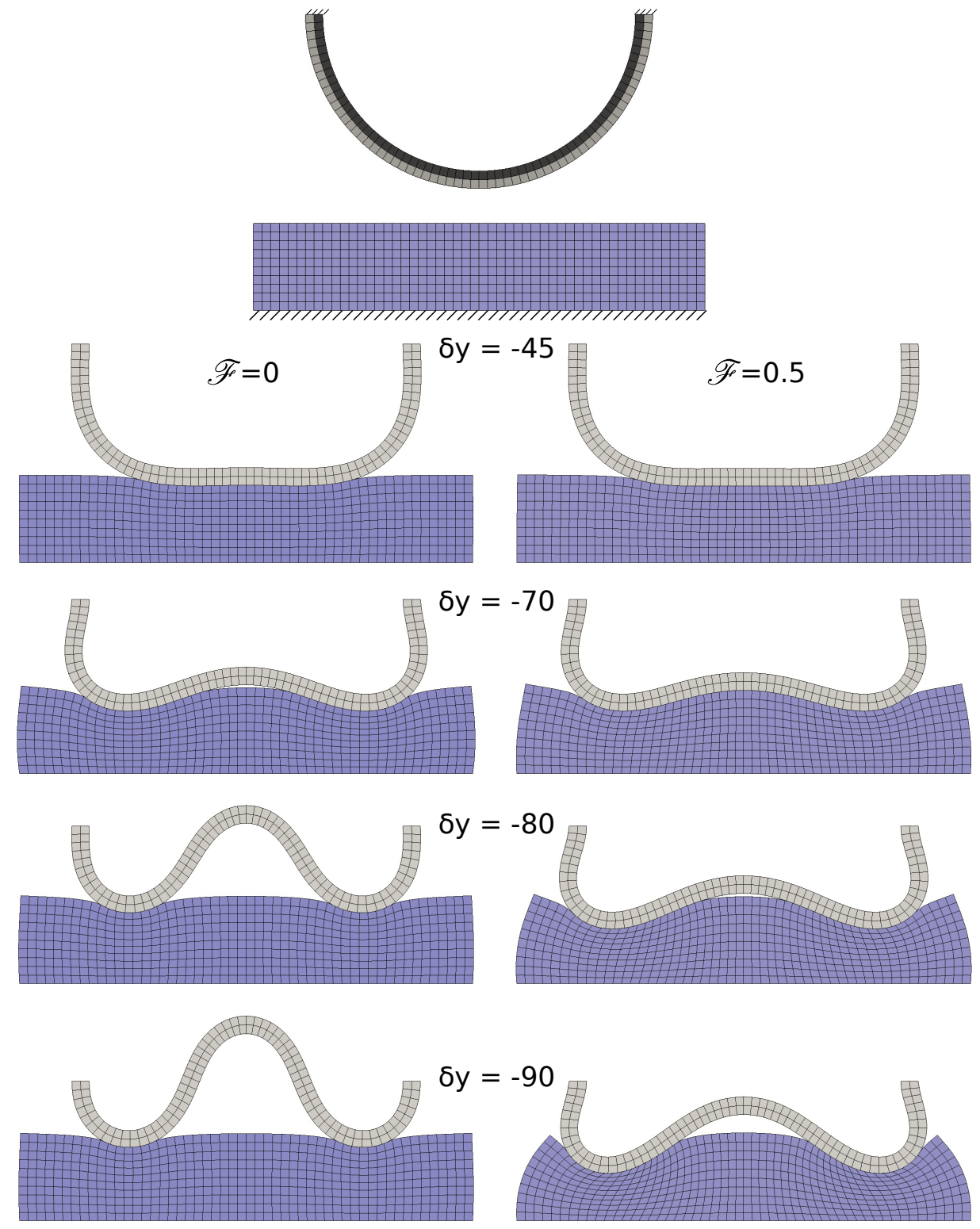

Figure 7: Initial and deformed configurations for the elastic half-ring on block problem at different simulation steps without and with friction.

Initially, the loaded half-ring compresses the rectangular block around a central contact spot, as expected. Beyond some limit though, both in the frictionless case and the case with a friction coefficient of 0.5 , the half-ring starts to fold and its middle point is lifted, resulting to two distinct contact spots. Fig. 8 shows the vertical displacement of the half-ring middle point during the simulation. Approximately up to the 50th simulation step, corresponding to the first deformed 
configuration in Fig. 7, the tracked middle point moves downwards as the rectangular block is being compressed. Subsequently, the tracked point is lifted progressively until the 100th simulation step, which corresponds to the second deformed configuration in Fig. 7. In the period between the 100th and the 120th simulation step, in absence of friction, the lifting speed of the half-ring middle point peaks. The ring is folded rapidly, while extensive sliding between the ring and the block occurs. In the presence of friction with a coefficient of 0.5 , sliding between the two parts is hindered and the tracked middle point appears to move upwards until the end of the simulation very progressively.

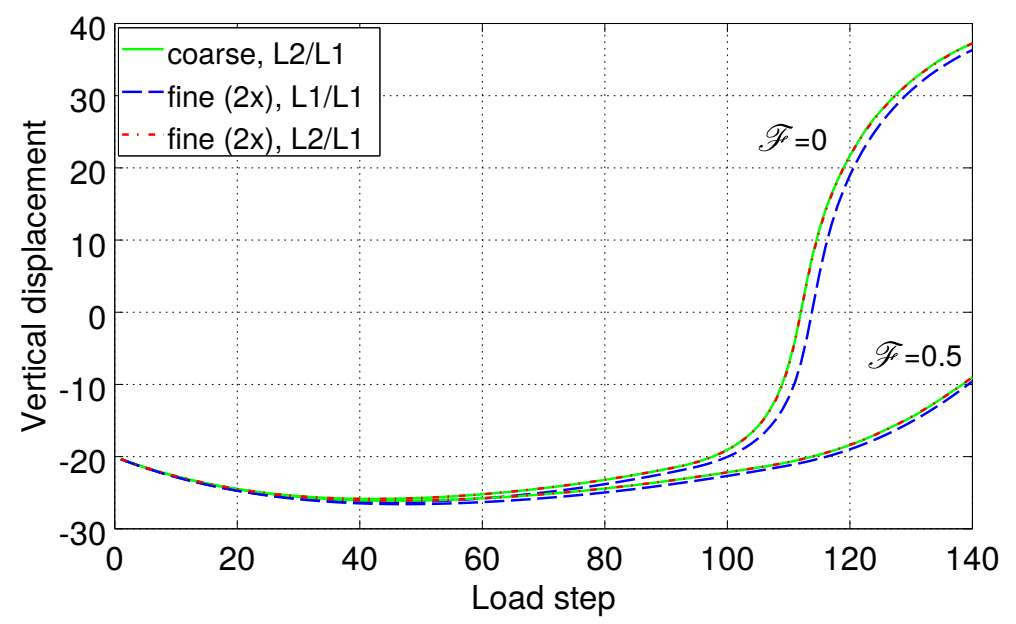

Figure 8: Vertical displacement of the half-ring middle point for different mesh sizes and finite element orders.

Apart from the combination of a second order approximation for the displacements and a first order approximation for the contact multiplier on the coarse mesh shown in Fig. 7, Fig. 8 also includes results from two further cases on a refined by a factor of two mesh. The second case presented in Fig. 8 is based on 4-node linear elements for the discretization of the displacements field, while the third case maintains the original 9-node quadratic elements for the displacements on the refined mesh. The first two cases have the same number of degrees of freedom, while the third case has the double amount of displacement degrees of freedom and is considered here as the most accurate approximation. A comparison between the first and the third case indicates that for 9-node second order elements the results obtained with the coarse and the refined mesh are very similar. On the contrary, refinement of the mesh by a factor of two combined with a corresponding reduction in the approximation order seems to provide less accurate results, presumably due to shear locking of the 4-node linear elements within the severely bended half-ring.

With respect to the numerical performance of the proposed contact formulation, Figures 9 and 10 show the number of Newton's iterations that are required in each simulation step, for reducing the initial 1-norm of the residuals vector by a factor of $10^{8}$ or more. The two figures contain results corresponding to the same cases as in Fig 8, with Fig 9 referring to the frictionless cases and Fig. 10 referring to the cases with friction. It appears that with the refined mesh and the inclusion of friction, the number of Newton's iterations increases only slightly. Moreover, the increased number of Newton's iterations observed in the period between the 100th and the 120th simulation step in the frictionless case, is less pronounced in the case with friction, due to the lack of considerable sliding. 
Other combinations of finite element orders, different numbers of quadrature points and interchanging slave and master surfaces are further possible options which are omitted here for the sake of space. It should be noted however, that applying a second order approximation of the contact multiplier did not result to any significant advantage for this specific example in terms of accuracy or convergence. Defining the block top edge as the slave surface instead of the ring outer edge resulted to a slightly improved convergence behavior.

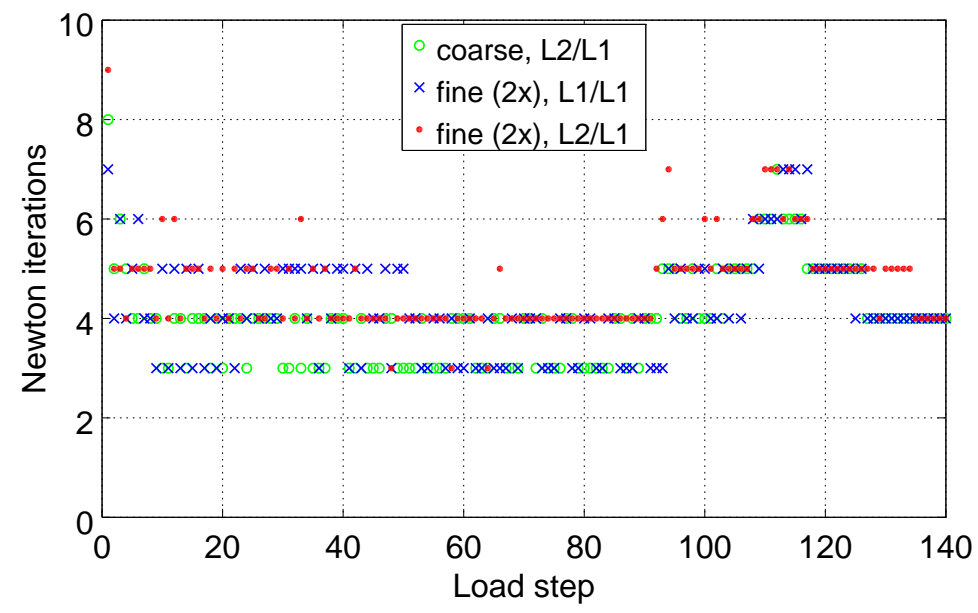

Figure 9: Number of required Newton's iterations per load step for different mesh sizes and finite element orders (frictionless).

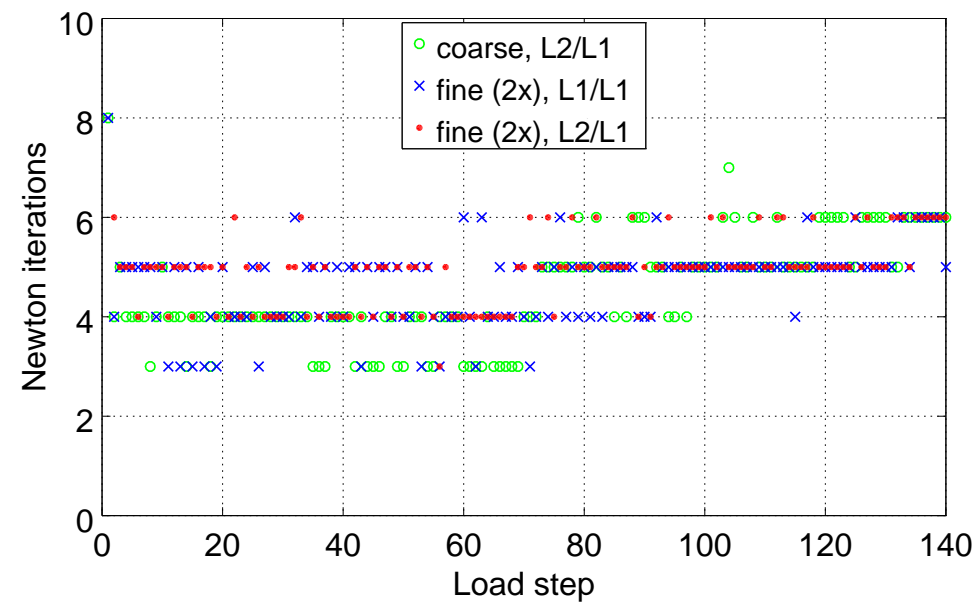

Figure 10: Number of required Newton's iterations per load step for different mesh sizes and finite element orders (friction coefficient of 0.5).

Specifically with respect to numerical integration in this example, an increased number of quadrature points eliminates occurrences of individual simulation steps exhibiting poor convergence. Alternatively, such occasional convergence issues could have been avoided also by incorporating a line-search step in the Newton's solution. However, we preferred to keep the unmodified Newton's method employing full steps for the sake of comparability of the presented examples. 


\subsection{Shallow ironing}

The third numerical example to be presented is the so-called shallow ironing problem. An indenter with a circular arc shaped bottom edge is pressed against a rectangular block and is forced to slide along the block length. This example can also be found, for instance, in [10] and [13]. Fig. 11 shows the initial and deformed geometry at different phases of the simulation. The contacting bodies exhibit, like in the previous example, neo-Hookean material behavior with Young's moduli equal to $68.96 \cdot 10^{8}$ and $68.96 \cdot 10^{7} \mathrm{MPa}$ for the indenter and the block respectively and Poisson's ratio of 0.32 for both parts. The considered two-dimensional system is solved under the plane strain assumption.
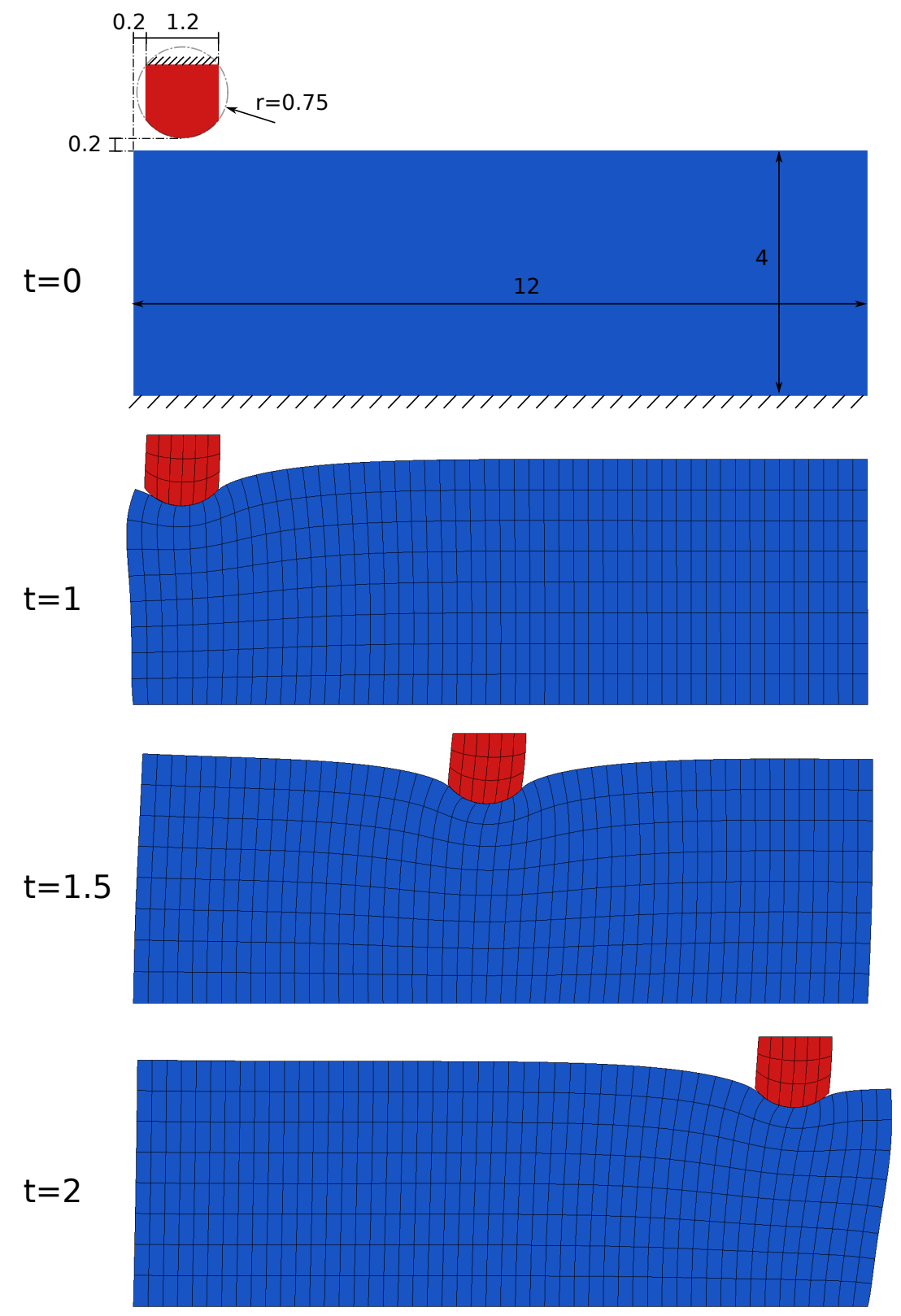

Figure 11: Initial and deformed configurations for the shallow ironing example with friction. 
Although the performed simulation is quasi-static, load steps are defined as a function of time for the sake of presentation of the results. From 0 to 1 second, the indenter is moved vertically towards the block by a total amount of $1 \mathrm{~mm}$ in 10 equal steps. From 1 to 2 seconds, the indenter is displaced horizontally by a total distance of $10 \mathrm{~mm}$ in 500 equal steps.

Fig. 12 shows the evolution of the total horizontal and vertical force components between the contacting bodies during the simulated period. Results, both for the frictionless case and for a friction coefficient of 0.3 , are included for comparison. The results presented in Figures 11 and 12 are based on a quadratic finite element approximation of the displacements field with 9-node quadrilateral elements and a linear approximation of the contact stress. Four quadrature points per segment are used for numerical integration on the indenter bottom edge which is defined as the slave surface.

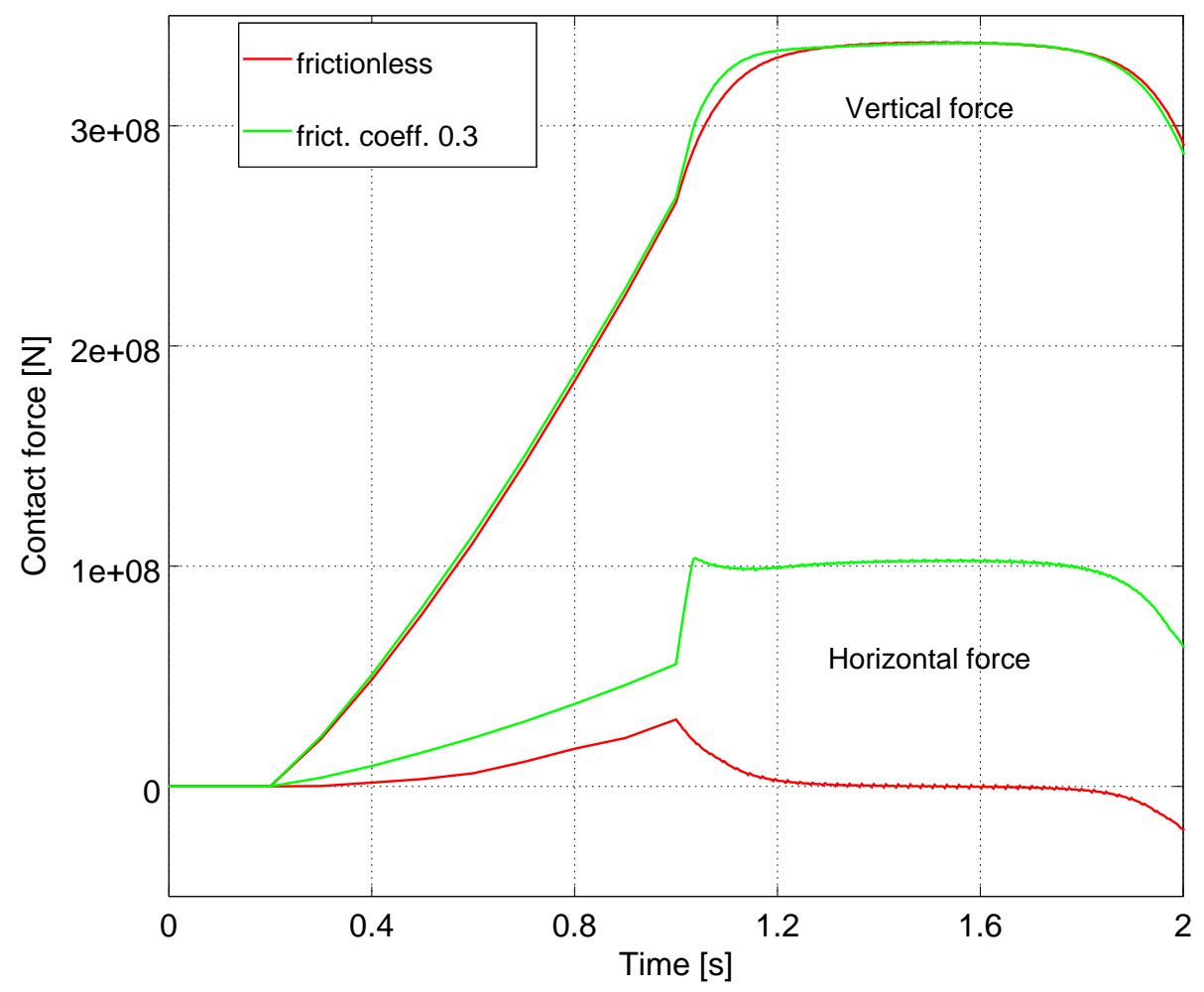

Figure 12: Calculated vertical and horizontal contact forces for the shallow ironing example with and without friction.

Regarding the case with friction, the curves shown in Fig. 12 exhibit the characteristic form found in previously published results [10, 13]. Nevertheless, there are important quantitative differences. The vertical force in Fig. 12 is slightly lower than in [10] and significantly lower than in [13], while the reported horizontal force is significantly lower compared to both aforementioned references. At time $1.5 \mathrm{~s}$ for instance, the ratio between the horizontal and vertical force can be estimated as 0.53, based on the results reported in [10] and 0.39 according to reference [13], while Fig. 12 corresponds to a ratio of only 0.304 . A similar ratio of 0.302 could be obtained by 
solving this example with the commercial finite element software Ansys ${ }^{\circledR}$. Despite the observed discrepancies, the here presented results appear to be plausible. Fig. 13 illustrates the calculated contact stresses field at time $1.5 \mathrm{~s}$. The contact stress distribution is according to common understanding of the system mechanics and the angle between the stress vectors and the surface normals appears to be very close to the friction angle of $16.7^{\circ}$ corresponding to the given coefficient of friction of 0.3. Moreover, for the frictionless case reported in Fig. 12, a zero horizontal force is predicted for the symmetric position at $1.5 \mathrm{~s}$, correctly.

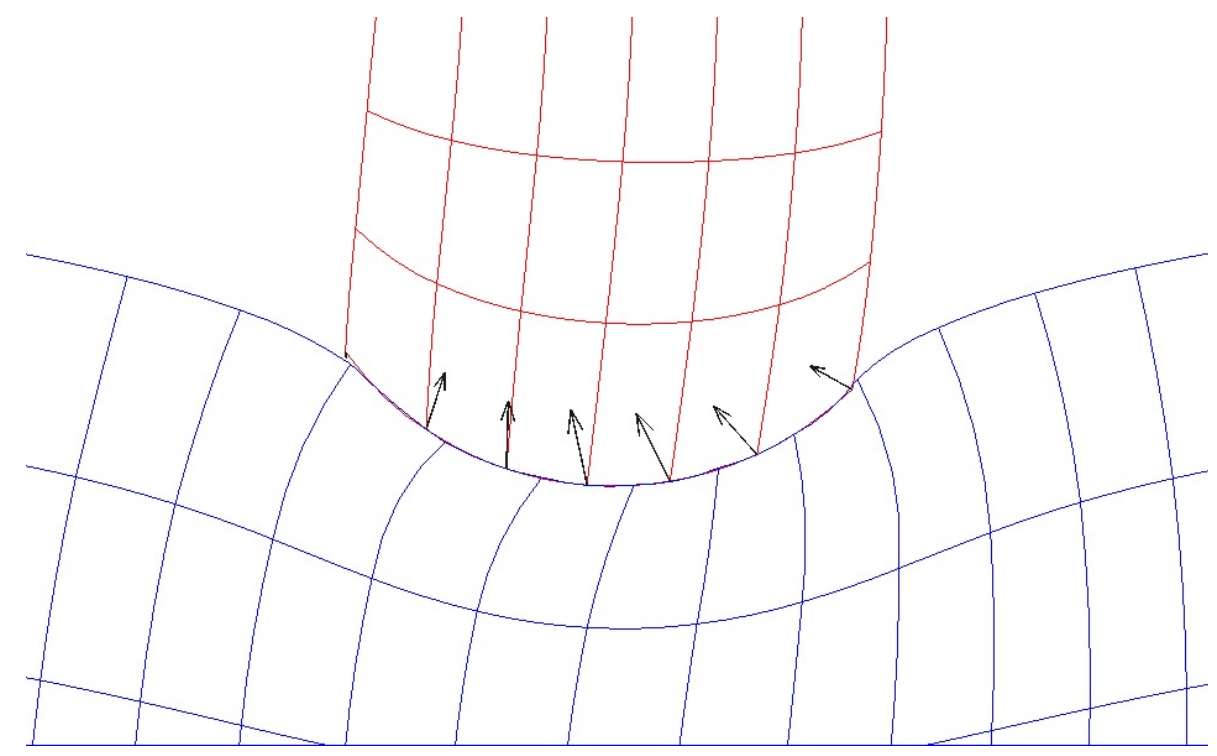

Figure 13: Calculated deformations and contact stresses at time $1.5 \mathrm{~s}$.

Results similar to those presented in Fig. 12 were obtained also with a finer mesh, different finite element approximations and different numbers of quadrature points. The average number of required Newton's iterations per load step in the period from 1 to 2 seconds remained, in all cases, approximately constant. Table 2 summarizes the average number of Newton's iterations per load step for different cases without and with friction. The first row of each table corresponds to one of the cases presented in Fig. 12 while each further row contains the parameters that differentiate every individual case from the first row.

In general, all reported cases deliver comparable convergence performance. The averagely required Newton's iterations per load step vary in the frictionless cases from 4.3 to 5.1, while in the cases with friction, 4.7 to 6.6 iterations are required in average. However, the accuracy of the result may depend significantly on the specific choice of solution parameters. As an example, Fig. 14 compares the contact forces calculated with three and four quadrature points per segment, for the case with friction and quadratic approximation of the displacements field. The solution with only three quadrature points per segment exhibits more pronounced oscillations of the calculated force, with wavelength equal to the mesh size. Nevertheless, the observed oscillations even with only three quadrature points are still relatively small, compared to similar results presented in [10] and [13]. 
Table 2: Average number of Newton's iterations from 1 to 2 seconds for different cases.

\begin{tabular}{|c|c|c|c|c|c|}
\hline \multicolumn{6}{|c|}{ Frictionless } \\
\hline $\begin{array}{l}\text { ñ } \\
\stackrel{0}{0} \\
\overrightarrow{0} \\
0 \\
0 \\
0 \\
\frac{1}{w} \\
\stackrel{0}{\Sigma}\end{array}$ & 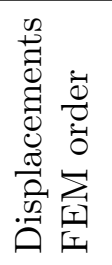 & 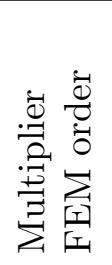 & 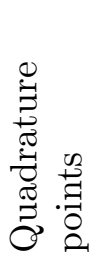 & 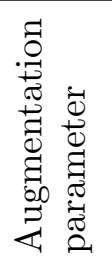 & 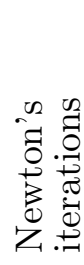 \\
\hline \multirow[t]{3}{*}{$1 x$} & 2 & 1 & 3 & $10^{3}$ & 4.7 \\
\hline & 1 & & & & 4.3 \\
\hline & & 2 & & & 5.1 \\
\hline $2 \mathrm{x}$ & 1 & & & & 5.0 \\
\hline \multirow[t]{7}{*}{$2 \mathrm{x}$} & & & & & 5.0 \\
\hline & 1 & & 4 & & 4.3 \\
\hline & & & 4 & & 4.6 \\
\hline & 1 & & 5 & & 4.3 \\
\hline & & & 5 & & 4.5 \\
\hline & & & & $10^{2}$ & 4.6 \\
\hline & & & & $10^{4}$ & 4.8 \\
\hline
\end{tabular}

\begin{tabular}{|c|c|c|c|c|c|c|}
\hline \multicolumn{7}{|c|}{ Friction coeff. equal to 0.3} \\
\hline 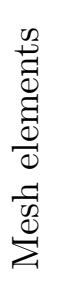 & 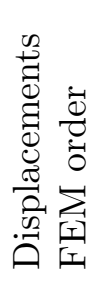 & 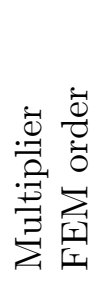 & 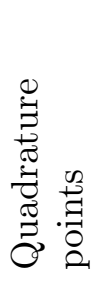 & 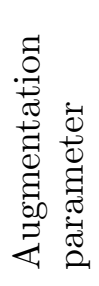 & 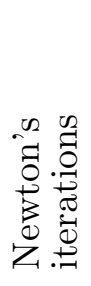 & 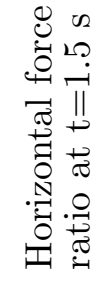 \\
\hline \multirow[t]{3}{*}{$1 \mathrm{x}$} & 2 & 1 & 3 & $10^{3}$ & 5.6 & 0.304 \\
\hline & 1 & & & & 5.0 & 0.281 \\
\hline & & 2 & & & 6.6 & 0.305 \\
\hline $2 \mathrm{x}$ & 1 & & & & 5.6 & 0.300 \\
\hline \multirow[t]{5}{*}{$2 \mathrm{x}$} & & & & & 6.1 & 0.300 \\
\hline & 1 & & 4 & & 4.7 & 0.273 \\
\hline & & & 4 & & 5.4 & 0.304 \\
\hline & 1 & & 5 & & 4.7 & 0.278 \\
\hline & & & 5 & & 5.4 & 0.303 \\
\hline & & & & $10^{2}$ & 5.1 & 0.293 \\
\hline & & & & $10^{4}$ & 5.7 & 0.302 \\
\hline
\end{tabular}
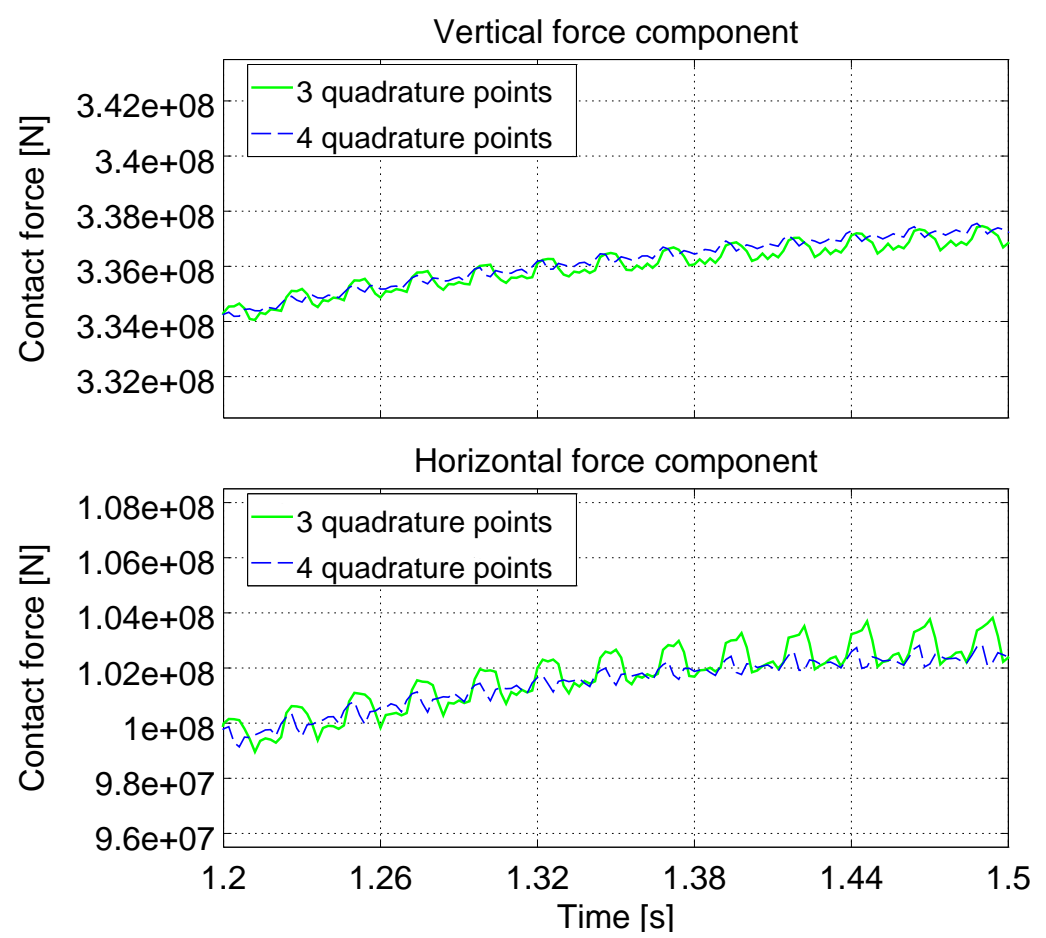

Figure 14: Calculated vertical and horizontal contact forces for the shallow ironing example with different numbers of quadrature points. 


\subsection{Crossed tubes}

The last numerical example refers to the contact between two crossed hollow cylinders. Each of these tubes has an outer diameter of $24 \mathrm{~mm}$, wall thickness equal to $0.8 \mathrm{~mm}$ and length equal to $100 \mathrm{~mm}$. Neo-Hookean material behavior is assumed for both tubes, with material parameters corresponding to Poisson's ratio equal to 0.3 as well as Young's moduli of $10^{5} \mathrm{MPa}$ for the lower tube and $10^{4} \mathrm{MPa}$ for the upper one.

The lower tube, oriented with its axis parallel to the $\mathrm{z}$ direction, and the upper tube, with its axis parallel to the $\mathrm{x}$ direction, are forced into contact through Dirichlet conditions applied on their free ends. Relatively to their initial configuration shown in Fig. 15, the ends of the lower tube remain fixed while the upper tube is displaced vertically. In a preliminary step, the upper tube is lowered by $20 \mathrm{~mm}$, corresponding to the initial gap between the tubes while in 80 further equal size steps, the ends of the upper tube are lowered by further $40 \mathrm{~mm}$.

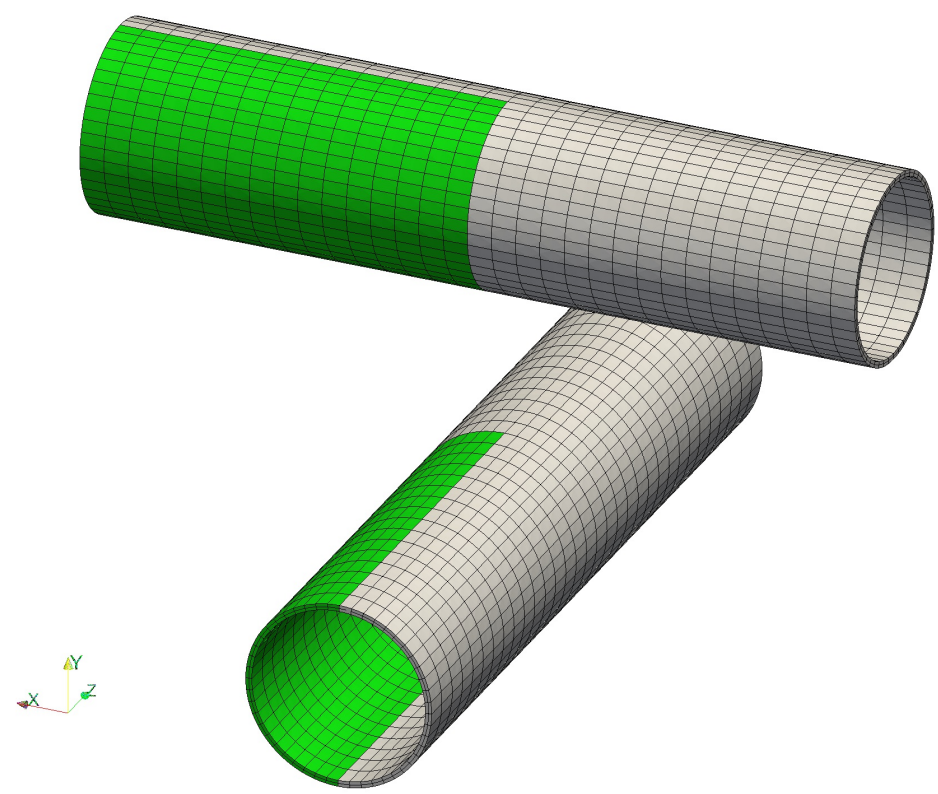

Figure 15: Geometry and mesh of the crossed tubes in their initial configuration.

Due to symmetric geometry and boundary conditions it is sufficient to model only one quarter of the considered structure. The actually modeled portion of each tube is identified in Fig. 15 in green color and is discretized with 16 by 24 by 2 three-dimensional elements in the length, circumferential and radial directions respectively. Fig. 16 shows the calculated deformed configurations for the 40th, 60th and 80th load steps. The presented solution is based on an approximation of the geometry and the displacements field with incomplete quadratic 20-node hexahedral elements. For the contact condition between the two tubes, the outer surface of the lower tube is considered as the slave surface. For the self-contact condition in the interior of the upper tube, its internal surface is considered as both slave and master surface at the same time. The contact stresses are approximated through linear elements on each of the slave surfaces, corresponding to faces of 8-node hexahedral elements. Numerical integration of contact terms is carried out based on sixteen quadrature points per element face. All reported results were obtained with an augmentation parameter of $10^{3} \mathrm{MPa} / \mathrm{mm}$. 

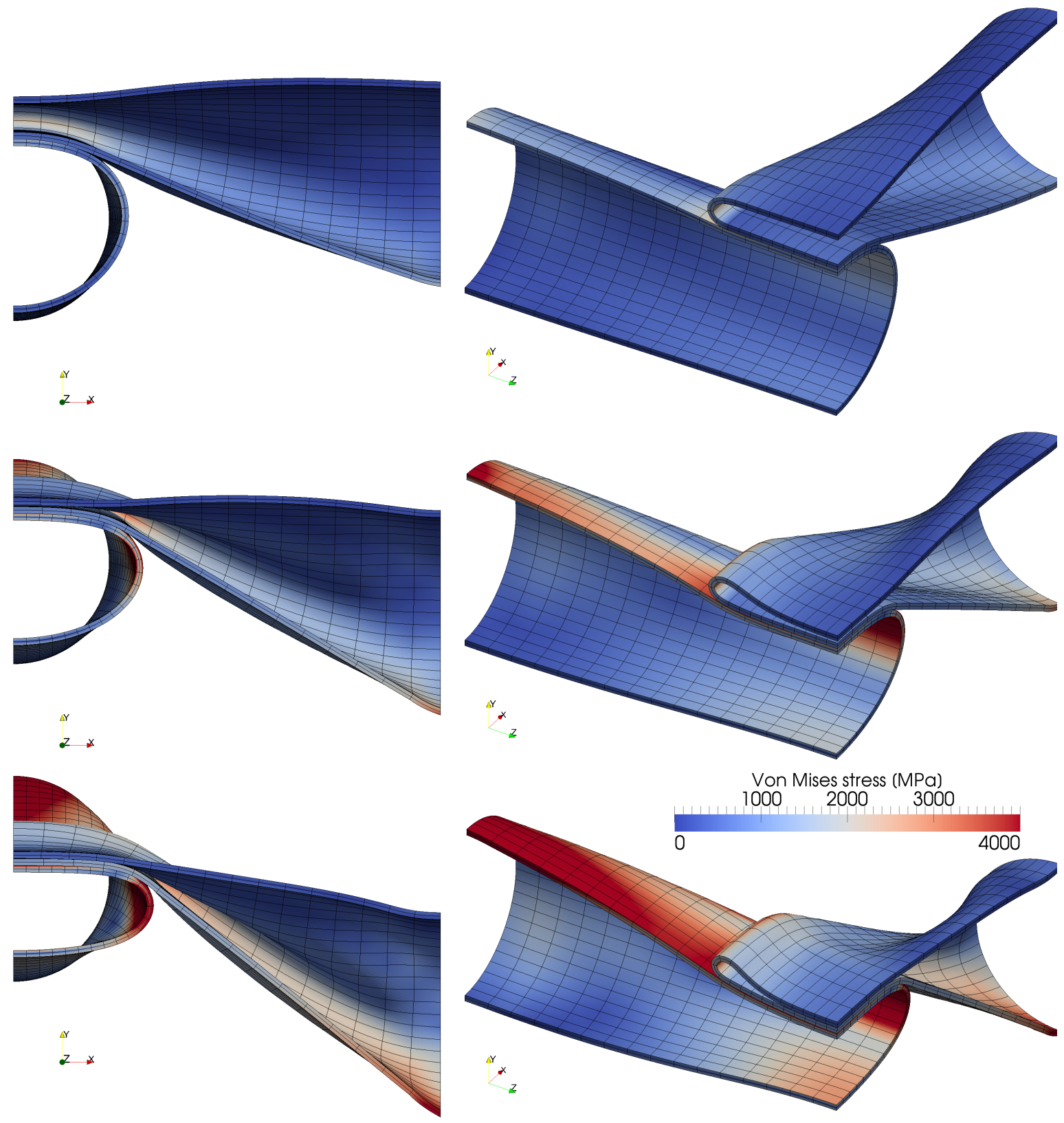

Figure 16: Deformed configurations of the crossed tubes in contact at 20, 30 and $40 \mathrm{~mm}$ of relative displacement between the tubes (top, middle and bottom respectively).

The results presented in Fig. 16 correspond to frictionless contact conditions, while Fig. 17 shows the required Newton's iterations for the frictionless case as well as for a friction coefficient of 0.3. Despite the lack of stabilization for dealing with a possible violation of the inf-sup condition related to the self-contact in the interior of the upper tube, Newton's algorithm converges in general fast. Only exception is the 62 nd load step, which actually corresponds to the onset of self contact in the interior of the upper tube. It is expected that applying an appropriate stabilization technique would help in achieving a good convergence at this single point as well. The relatively 
good performance observed after the 62nd load step, despite the lack of stabilization, can possibly be attributed to the good alignment between the contacting surface mesh portions in the interior of the upper tube, due to the symmetry in this specific example.

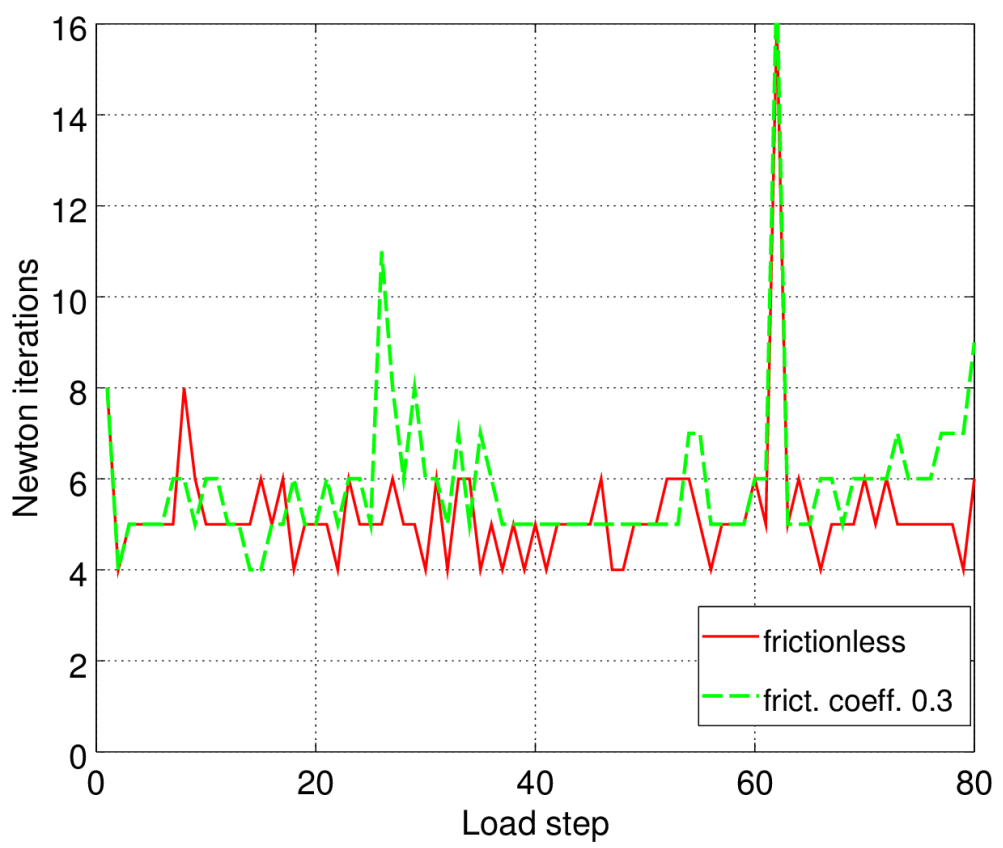

Figure 17: Number of required Newton's iterations per load step without and with friction.

\section{Concluding remarks}

In this paper, we have presented an integral approximation of frictional contact between deformable bodies under finite deformations and large sliding. The distinguishing characteristic of the proposed formulation is that it enforces complementarity conditions in a weak sense. As a consequence, it does not require contact and non-contact or slip and stick regions to be defined through active and inactive sets of finite element nodes, leading to the following two advantages:

- Reduced oscillations arising from finite element nodes entering the contact region and shifting between stick and slip regions. Moreover, as shown in the shallow ironing example, such oscillations vanish with increasing number of quadrature points.

- Although the numerical examples shown here do not cover such cases, the proposed method is directly applicable to non-Lagrange finite element approximations, such as isogeometric or enriched elements.

Another important characteristic of the proposed formulation is the definition of the contact normal direction according to the slave surface. Although this choice is very common in mortar contact formulations, here it has been justified through a comprehensive comparison with the classical projection strategy. In order to clearly identify the two possible options of defining the contact normal either according to the master or the slave surface, the term "ray-tracing" 
has been introduced for describing the gap definition in the latter case as the counterpart of the classical term "projection" referring to the first case. We hope that introduction of the term "ray-tracing" will allow to distinguish between the two aforementioned options in the future more clearly and to evaluate their comparative advantages and disadvantages purposefully.

In total, combining an integral enforcement of a non-smooth complementarity equation with the ray-tracing strategy has resulted to: 1) a system Lagrangian function of enhanced regularity with respect to the deformation, 2) a relatively simple tangent system and 3) compatibility with non-Lagrange finite element approximations. The simplicity of the formulation consists in the tangent system not depending on the curvature of the contact surfaces and geometry corners not requiring any special treatment.

The presented two-dimensional examples have revealed some practical aspects and demonstrated the robustness of the proposed formulation. The Hertzian contact example showed the importance of choosing an augmentation parameter based on the elasticity modulus of the contacting bodies and some characteristic length in the same order of magnitude as the contact width. Moreover, it showed a very good approximation of the pressure profile when quadratic elements are used both for the displacements and the contact stress fields. Nevertheless, this combination of second order elements requires that numerical integration is performed with at least three quadrature points per contact element. The elastic half-ring and shallow ironing examples showed that the proposed method required a relatively low number of Newton's iterations, even for problems with a considerable amount of sliding per load step. In general, four to five Newton's iterations per load step were sufficient in the frictionless case, while five to six iterations were typically required in the presence of friction. Moreover, refining the mesh appears to affect the number of required Newton's iterations only slightly. It should be noted that eight quadrature points per contact element ensured good convergence in the elastic half-ring example, while only four quadrature points per contact element were sufficient in the shallow ironing example for achieving satisfactory results both in terms of accuracy and convergence.

The example of contact between two crossed tubes has demonstrated a comparable performance in three dimensions like in the two-dimensional examples. In general, four to six Newton's iterations per load step were required for the case without friction, while the case with friction required slightly more iterations averagely. The crossed tubes example also included a self-contact situation, where both sides of the contact interface are slave and master surfaces at the same time. Despite the lack of any special treatment for this situation which violates the inf-sup condition between the contact stress and displacements approximations, this numerical experiment indicated poor convergence only during a single load step corresponding to the onset of self-contact. Extending the proposed formulation through an appropriate stabilization technique for dealing with violation of the inf-sup condition is reserved for future work.

\section{Appendix A: Partial derivatives of $\mathcal{C}(\lambda, g, \mathrm{v}, n)$}

Making use of the Heaviside function, defined as:

$$
H(x)=\left\{\begin{array}{l}
0, \text { for } x<0 \\
1, \text { for } x \geq 0
\end{array}\right.
$$


partial derivatives of quantity $\tau=\mathscr{F}[\lambda \cdot n+r g]_{-}$can be expressed as:

$$
\begin{aligned}
\partial_{\lambda} \tau & =-H(-\lambda \cdot n-r g) \mathscr{F} n, \\
\partial_{g} \tau & =-H(-\lambda \cdot n-r g) \mathscr{F} r, \\
\partial_{n} \tau & =-H(-\lambda \cdot n-r g) \mathscr{F} \mathbf{T}_{n} \lambda .
\end{aligned}
$$

Moreover, writing $q_{T}=\mathbf{T}_{n} q$ for simplicity, partial derivatives of projection $P_{B(n, \tau)}(q)$ can be expressed as:

$$
\begin{aligned}
& \partial_{q} P_{B(n, \tau)}(q)=\left\{\begin{array}{cl}
0 & \text { for } \tau \leq 0 \\
\mathbf{T}_{n} & \text { for }\left\|q_{T}\right\| \leq \tau \\
\frac{\tau}{\left\|q_{T}\right\|}\left(\mathbf{T}_{n}-\frac{q_{T}}{\left\|q_{T}\right\|} \otimes \frac{q_{T}}{\left\|q_{T}\right\|}\right) & \text { otherwise }
\end{array}\right. \\
& \partial_{\tau} P_{B(n, \tau)}(q)=\left\{\begin{array}{cl}
0 & \text { for } \tau \leq 0 \text { or }\left\|q_{T}\right\| \leq \tau \\
\frac{q_{T}}{\left\|q_{T}\right\|} & \text { otherwise }
\end{array}\right. \\
& \partial_{n} P_{B(n, \tau)}(q)=\left\{\begin{array}{cl}
0 & \text { for } \tau \leq 0 \\
-q \cdot n \mathbf{T}_{n}-n \otimes q_{T} & \text { for }\left\|q_{T}\right\| \leq \tau \\
-\frac{\tau}{\left\|q_{T}\right\|}\left(q \cdot n\left(\mathbf{T}_{n}-\frac{q_{T}}{\left\|q_{T}\right\|} \otimes \frac{q_{T}}{\left\|q_{T}\right\|}\right)+n \otimes q_{T}\right) & \text { otherwise. }
\end{array}\right.
\end{aligned}
$$

Finally, the partial derivatives of function $\mathcal{C}$ can be calculated as:

$$
\begin{aligned}
\partial_{\lambda} \mathcal{C}(\lambda, g, \mathrm{v}, n)= & \mathbf{I}-\partial_{q} P_{B(n, \tau)}-\partial_{\tau} P_{B(n, \tau)} \otimes \partial_{\lambda} \tau-H(-\lambda \cdot n-r g) n \otimes n, \\
\partial_{g} \mathcal{C}(\lambda, g, \mathrm{v}, n)= & -\partial_{\tau} P_{B(n, \tau)} \partial_{g} \tau-H(-\lambda \cdot n-r g) r n, \\
\partial_{n} \mathcal{C}(\lambda, g, \mathrm{v}, n)= & -\partial_{n} P_{B(n, \tau)}-\partial_{\tau} P_{B(n, \tau)} \otimes \partial_{n} \tau \\
& -H(-\lambda \cdot n-r g)(n \otimes \lambda-(2 \lambda \cdot n+r g) n \otimes n+(\lambda \cdot n+r g) \mathbf{I}), \\
\partial_{\mathrm{v}} \mathcal{C}(\lambda, g, \mathrm{v}, n)= & r \partial_{q} P_{B(n, \tau)},
\end{aligned}
$$

where the argument $q=\lambda-r \mathrm{v}$ of the projection $P_{B(n, \tau)}(q)$, found in the definition of $\mathcal{C}$, is omitted in the presentation of the partial derivatives of $\mathcal{C}$ for the sake of brevity.

\section{References}

[1] P. Alart, A. Curnier. A generalized Newton method for contact problems with friction. J. Mech. Theor. Appl., 7:1 (1988), 67-82.

[2] P.R. Amestoy, A. Guermouche J.-Y. L'Excellent, S. Pralet. Hybrid scheduling for the parallel solution of linear systems. Parallel Computing, 32:2 (2006), 136-156.

[3] K-J. Bathe, F. Brezzi. Stability of finite element mixed interpolations for contact problems. Atti Accad. Naz. Lincei Cl. Sci. Fis. Mat. Natur. Rend. Lincei (9) Mat. Appl., 12:3 (2001), 167-183.

[4] F. Ben Belgacem, Y. Renard. Hybrid finite element methods for the Signorini problem. Math. Comp., 72:243 (2003), 1117-1145. 
[5] A. Curnier, Q.C. He, A. Klarbring. Continuum mechanics modelling of large deformation contact with friction. Contact Mechanics, (1995), 145-158.

[6] L. De Lorenzis, P. Wriggers, G. Zavarise. A mortar formulation for 3D large deformation contact using NURBS-based isogeometric analysis and the augmented Lagrangian method. Comput. Mech., 49 (2012), 1-20.

[7] N. El-Abbasi, K-J. Bathe. Stability and patch test performance of contact discretizations and a new solution algorithm. Comput. Struct., 79 (2001), 1473-1486.

[8] A.L. Eterovic, K-J. Bathe. On the treatment of inequality constraints arising from contact conditions in finite element analysis. Comput. Struct., 40:2 (1991), 203-209.

[9] K.A. Fischer, P. Wriggers. Frictionless 2D contact formulations for finite deformations based on the mortar method. Comput. Mech., 36 (2005), 226-244.

[10] K.A. Fischer, P. Wriggers. Mortar based frictional contact formulation for higher order interpolations using the moving friction cone. Comput. Methods Appl. Mech. Engrg., 195 (2006), 5020-5036.

[11] M. Gitterle, A. Popp, M.W. Gee, and W.A. Wall. Finite deformation frictional mortar contact using a semi-smooth Newton method with consistent linearization. Int. J. Numer. Meth. Engng., 84 (2010), 543-571.

[12] G. Haikal, K.D. Hjelmstad. A finite element formulation of non-smooth contact based on oriented volumes for quadrilateral and hexahedral elements. Comput. Methods Appl. Mech. Engrg., 196 (2007), 4690-4711.

[13] S. Hartmann, J. Oliver, R. Weyler, J.C. Cante, J.A. Hernández. A contact domain method for large deformation frictional contact problems. Part 2: Numerical aspects. Comput. Methods Appl. Mech. Engrg., 198 (2009), 2607-2631.

[14] P. Hild, P. Laborde. Quadratic finite element methods for unilateral contact problems. Appl. Numer. Math., 41 (2002), 420-421.

[15] M. Hintermüller, K. Ito, K. Kunisch. The primal-dual active set strategy as a semismooth Newton Method. SIAM J. Optim., 13:3 (2003), 865-888.

[16] A. Konyukhov, K. Schweizerhof. Computational Contact Mechanics. Lecture Notes in Applied and Computational Mechanics, vol. 67, Springer-Verlag, Berlin 2013.

[17] P. Laborde, Y. Renard. Fixed point strategies for elastostatic frictional contact problems. Math. Meth. Appl. Sci., 31 (2008), 415-441.

[18] T.A. Laursen, J.C. Simo. A Continuum-Based Finite Element Formulation for the Implicit Solution of Multibody, Large Deformation Frictional Contact Problems. Int. J. Numer. Meth. Engng., 36 (1993), 3451-3485.

[19] T.W. McDevitt, T.A. Laursen. A mortar-finite element formulation for frictional contact problems. Int. J. Numer. Meth. Engng., 48 (2000), 1525-1547.

[20] J. Oliver, S. Hartmann, J.C. Cante, R. Weyler, J.A. Hernández. A contact domain method for large deformation frictional contact problems. Part 1: Theoretical basis. Comput. Methods Appl. Mech. Engrg., 198 (2009), 2591-2606.

[21] D. Pantuso, K.-J. Bathe, P.A. Bouzinov. A finite element procedure for the analysis of thermo-mechanical solids in contact. Comput. Struct., 75:6 (2000), 551-573. 
[22] J. Pommier, Y. Renard. GetFEM++, an open source generic C++ library for finite element methods. http://download.gna.org/getfem/html/homepage/.

[23] M.A. Puso, T.A. Laursen. A 3D contact smoothing method using Gregory patches. Int. J. Numer. Meth. Engng., 54 (2002), 1161-1194.

[24] M.A. Puso, T.A. Laursen. A mortar segment-to-segment frictional contact method for large deformations. Comput. Methods Appl. Mech. Engrg., 193 (2004), 4891-4913.

[25] Y. Renard. Generalized Newton's methods for the approximation and resolution of frictional contact problems in elasticity. Comput. Methods Appl. Mech. Engrg., 256 (2013), 38-55.

[26] J.C. Simo, T.A. Laursen. An augmented Lagrangian treatment of contact problems involving friction. Comput. Struct., 42:1 (1992), 97-116.

[27] I. Temizer, P. Wriggers, T.J.R. Hughes. Contact treatment in isogeometric analysis with NURBS Comput. Methods Appl. Mech. Engrg., 200 (2011), 1100-1112.

[28] M. Tur, F.J. Fuenmayor, P. Wriggers. A mortar-based frictional contact formulation for large deformations using Lagrange multipliers Comput. Methods Appl. Mech. Engrg., 198 (2009), 2860-2873.

[29] S.P. Wang, E. Nakamachi. The inside-outside contact search algorithm for finite element analysis. Int. J. Numer. Meth. Engng., 40 (1997), 3665-3685.

[30] P. Wriggers. Finite Element Algorithms for Contact Problems. Arch. Comput. Method. E., 2:4 (1995), 1-49.

[31] P. Wriggers, G. Zavarise. A formulation for frictionless contact problems using a weak form introduced by Nitsche. Comput. Mech., 41 (2008), 407-420.

[32] P. Wriggers, J. Schröder, A. Schwarz. A finite element method for contact using a third medium. Comput. Mech., 52:4 (2013), 837-847.

[33] B. Yang, T.A. Laursen, X. Meng. Two dimensional mortar contact methods for large deformation frictional sliding. Int. J. Numer. Meth. Engng., 62 (2005), 1183-1225.

[34] G. Zavarise, P. Wriggers. A Segment-to-Segment Contact Strategy. Mathl. Comput. Modelling, 28:4-8 (1998), 497-515. 